Berkeley Law

From the SelectedWorks of Aaron Edlin

December, 1998

\title{
Is Perfect Price Discrimination Really Efficient? Welfare and Existence in General Equilibrium
}

Aaron S. Edlin

Mario Epelbaum

Walter P. Heller 


\title{
IS PERFECT PRICE DISCRIMINATION REALLY EFFICIENT?: WELFARE AND EXISTENCE IN GENERAL EQUILIBRIUM
}

\author{
By Aaron S. Edlin, Mario Epelbaum, and Walter P. Heller ${ }^{1}$
}

\begin{abstract}
We examine the welfare properties of surplus maximization by embedding a perfectly discriminating monopoly in an otherwise standard Arrow-Debreu economy. Although we discover an inefficient equilibrium, we validate partial equilibrium intuition by showing: (i) that equilibria are efficient provided that the monopoly goods are costly, and (ii) that a natural monopoly can typically use personalized two-part tariffs in these equilibria. However, we find that Pareto optima are sometimes incompatible with surplus maximization, even when transfer payments are used. We provide insight into the source of this difficulty and give some instructive examples of economies where a second welfare theorem holds.
\end{abstract}

KEYwORDS: Price discrimination, surplus maximization, regulation, general equilibrium.

\section{INTRODUCTION}

SOME ECONOMISTS ARGUE that the production of natural monopolies should be regulated, while others, such as Demsetz (1968), argue that publicly owned or regulated firms waste resources, and so monopoly rights should be auctioned. Both groups agree, however, that the correct social goal for the natural monopoly is to maximize the sum of producer and consumer surplus in its market.

This paper explores whether this consensus is warranted. To do so, we examine the welfare consequences of an idealized perfectly discriminating monopoly (PDM), and ask if anything is lost when it maximizes surplus in its own market without regard to other markets. The efficiency of perfect price discrimination is considered well-established, but we note that the existing proofs typically use partial equilibrium arguments that often depend upon convexity and that do not account for interactions with other markets or for the circular flow of income.

These arguments leave open questions about both efficiency and distribution. For instance, can inefficiency arise from a PDM destroying surplus in another market in the course of creating surplus in its own, as can happen in monopolis-

\footnotetext{
${ }^{1}$ We appreciate the comments of an editor, three excellent referees, Donald Brown, Alejandro Hernández, Mordecai Kurz, Paul Romer, Manuel Santos, Christina Shannon, David Starrett, Joseph Stiglitz, and the participants in various seminars where we presented this paper. We thank Eric Emch for his valuable research assistance. Aaron Edlin thanks the Centro de Investigación Económica, Instituto Tecnológico Autónomo de México for supporting part of this work during a visit in October 1993, the National Science Foundation for support during graduate school, and the Hoover Institution at Stanford University for a Hoover National Fellowship during 1996-97. The authors themselves are solely responsible for the views expressed and any errors.
} 
tic competition? ${ }^{2}$ Or, leaving efficiency aside, does surplus maximization in a single market limit over-all distributional flexibility? Such questions create a call for a general equilibrium analysis of perfect price discrimination under increasing returns, particularly given the many examples of inefficiencies and decentralization problems found in the recent increasing returns literature. ${ }^{3}$

This paper therefore introduces a new general equilibrium concept-a perfectly discriminating monopoly equilibrium. Our economy has many price-taking firms that produce competitive goods sold at linear prices. There is also a PDM that produces monopoly goods, and that uses or produces these competitive goods. The PDM treats household incomes and the prices of competitive goods as constant, and sets individualized nonlinear tariffs for the monopoly goods in order to maximize profits. These profits are measured in accounting units whose value is tied down by the equilibrium prices of competitive goods. Households receive incomes calculated according to equilibrium prices and profits, and just as in an Arrow-Debreu general equilibrium, they maximize utility treating their incomes and the prices they pay as constant.

The fact that our PDM treats incomes and some prices as fixed differs from many general equilibrium models of imperfect competition in which large firms treat little or nothing as fixed (see, e.g., Gabszewicz and Vial (1972), Grodal (1984), and Böhm (1994)). In many cases, our approach seems realistic: for example, a local telephone company may set prices in telephone service, but take the price of labor or telephone wire as given. Our approach also has a methodological justification: in order to better understand the impact of industry surplus maximization on related markets and to test the robustness of partial equilibrium analysis, it makes sense not to abandon all of the partial equilibrium assumptions at once. An incidental advantage of our approach is that it removes the dependence of equilibria on the normalization rule.

Our analysis reveals that it is possible for a PDM equilibrium to be inefficient, but that all PDM equilibria are efficient when the monopoly goods are costly. On the other hand, we find that some optima cannot be decentralized when the monopoly has increasing returns. This reveals a tension between surplus maximization and fulfilling certain social objectives. Decentralization can fail for reasons other than the lack of sufficient willingness-to-pay found in the regulatory literature on two-part tariffs (see Vohra (1990), Quinzii (1992), and Brown, Heller, and Starr (1992)). Too much willingness-to-pay at other allocations can

${ }^{2}$ In monopolistic competition with linear pricing, Spence (1976) writes that,

... when a product is introduced it affects other firms' profits... Since the entering firm does not take these effects into account, it may enter when it is not generating a net social benefit.

\footnotetext{
${ }^{3}$ For examples of inefficiencies under increasing returns with first-order conditions satisfied, see Guesnerie (1975), Brown and Heal (1979), Beato and Mas-Colell (1983), or more recently Vohra (1990) or Brown, Heller, and Starr (1992). For an old discussion of some such inefficiencies see Coase (1946). For a discussion of decentralization failures, see Vohra (1990), Quinzii (1992), and Brown, Heller, and Starr (1992).
} 
be equally problematic. Our example of a Pareto optimum that cannot be decentralized also serves to show that a PDM equilibrium sometimes will not exist. This fact should not lessen interest in our equilibrium concept, since as mentioned above, our goal is to test the robustness of partial equilibrium models of surplus maximization, and a nonexistence result reveals a lack of robustness.

After Section 3 presents the above results, Section 4 improves our geometric and economic understanding by characterizing the sets of production plans that yield the monopoly the same or more profit than in some candidate for equilibrium. This analysis reveals that problems with decentralization arise when increasing returns are too dramatic. It also helps us show that a PDM can generally use two-part tariffs, charging a uniform per-unit price equal to marginal cost and discriminating by varying hookups among buyers. Section 5 explores two special cases in which Pareto optima can be decentralized and equilibria exist - economies with a single competitive good and economies with a pure fixed cost. Finally, Section 6 compares our equilibrium notion with the literature.

\section{THE MODEL}

\subsection{Primitives}

The economy has $H$ households, $F-1$ competitive firms, 1 monopoly firm, $m$ monopoly goods, and $c$ competitive goods. The competitive firms $f \neq m$ have closed convex production sets denoted $\mathbf{Y}^{f} \subset \Re^{m+c}$, with $\mathbf{0} \in \mathbf{Y}^{f}$; they cannot produce the first $m$ goods, so $\mathbf{Y}^{f} \subset \mathfrak{R}_{-}^{m} \times \mathfrak{R}^{c}$. Firm $m$ is a monopoly firm with the exclusive ability to produce the first $m$ goods. Its production possibility set is $\mathbf{Y}^{m} \subset \Re_{+}^{m} \times \Re^{c}$, with $\mathbf{0} \in \mathbf{Y}^{m}$; this set may be nonconvex and exhibiting increasing returns. Each production set $\mathbf{Y}^{f}$ is the free disposal hull of some compact set $\mathbf{K}^{f}$, so that $\mathbf{Y}^{f} \equiv \mathbf{K}^{f}-\Re_{+}^{m+c}$. . We assume that $\sum_{f=1}^{F} \mathbf{Y}^{f} \cap \Re_{+}^{m+c}=\{\mathbf{0}\}$. The monopoly firm can produce or use any of the $c$ competitive goods and the competitive firms may use the $m$ monopoly goods as inputs.

Each household $h$ has a continuous, strictly monotonic, quasi-concave utility function $U^{h}: \Re_{+}^{m+c} \rightarrow \Re$, owns a share $\theta^{h f} \geq 0$ of each firm $f$, and is endowed with $\boldsymbol{\omega}^{h} \in \Re_{+}^{m+c}$. Naturally, $\sum_{h} \theta^{h f}=1$ for all $f$. Finally, we assume that households are not endowed with the monopoly goods, so $\forall h, \boldsymbol{\omega}^{h}=\left(\boldsymbol{0}_{m}, \boldsymbol{\omega}_{c}^{h}\right)$, where $\boldsymbol{\omega}_{c}^{h} \in \Re_{+}^{c}$.

The economy $E$ is defined by $E \equiv\left\langle\left(\boldsymbol{\omega}^{h}\right),\left(U^{h}\right),\left(\mathbf{Y}^{f}\right),\left(\theta^{h f}\right)\right\rangle$.

Our convention is that vectors (lists) are always in boldface. The $i$ th component of a vector is not boldfaced, so the $i$ th component of the vector $\mathbf{y}^{f}$ is the scalar $y_{i}^{f}$. We partition vectors of prices or of goods using the subscript " $m$ " for the first $m$ components, and the subscript " $c$ " for the last $c$ components, so that firm $f$ 's production plan is partitioned as follows: $\mathbf{y}^{f}=\left(\mathbf{y}_{m}^{f}, \mathbf{y}_{c}^{f}\right)$. Similarly, we

\footnotetext{
${ }^{4}$ This assumption ensures that the attainable sets are compact, following Beato and Mas-Colell (1985) and Vohra (1992, p. 865). We do not require this assumption for our main results in Sections 3 and 4, but do require it for the special cases in Section 5 .
} 
write interchangeably $U^{h}\left(\mathbf{x}^{h}\right)$ and $U^{h}\left(\mathbf{x}_{m}^{h}, \mathbf{x}_{c}^{h}\right)$. We denote a list of vectors with parentheses, usually writing $\left(\mathbf{x}^{h}\right)$ instead of $\left(\mathbf{x}^{h}\right)_{h=1}^{H}$, and we write their sum by omitting the superscript, so that $\mathbf{x} \equiv \sum_{h} \mathbf{x}^{h}, \boldsymbol{\omega} \equiv \sum_{h} \boldsymbol{\omega}^{h}$, and $\mathbf{Y} \equiv \sum_{f} \mathbf{Y}^{f}$. Finally, we write $\mathbf{a} \leq \mathbf{b}$ if $a_{i} \leq b_{i} \forall i ; \mathbf{a}<\mathbf{b}$ if $a_{i} \leq b_{i} \forall i$ and $a_{j}<b_{j}$ for some $j$; and $\mathbf{a} \ll \mathbf{b}$ if $a_{i}<b_{i} \forall i$.

\subsection{The Perfectly Discriminating Monopoly}

The PDM maximizes profits by offering each household or firm an individualized pricing rule that maps the amount that buyer purchases into a total charge. In principle, a given offer might be an arbitrary function $g: \Re_{+}^{m} \rightarrow \Re$, where $g\left(\mathbf{0}_{m}\right) \leq 0$, giving the total charge for any bundle of monopoly goods. However, a perfect price discriminator need only make take-it-or-leave-it offers.

The following definitions permit a simple statement of the PDM's problem. Let $\mathbf{p}_{c} \gg \mathbf{0}_{c}$ be the competitive prices. A household $h$ consuming $\mathbf{x}_{m}^{h} \geq \mathbf{0}_{m}$ of the monopoly goods and spending $I$ on the competitive goods has utility

$$
V^{h}\left(\mathbf{x}_{m}^{h}, I, \mathbf{p}_{c}\right) \equiv \max _{\mathbf{x}_{c}} U^{h}\left(\mathbf{x}_{m}^{h}, \mathbf{x}_{c}\right) \quad \text { subject to } \quad \mathbf{p}_{c} \cdot \mathbf{x}_{c} \leq I .
$$

Similarly, let

$$
\Pi^{f}\left(\mathbf{y}_{m}^{f}, \mathbf{p}_{c}\right) \equiv \max _{\mathbf{y}_{c}} \mathbf{p}_{c} \cdot \mathbf{y}_{c} \quad \text { subject to } \quad\left(\mathbf{y}_{m}^{f}, \mathbf{y}_{c}\right) \in \mathbf{Y}^{f}, \quad f \neq m .
$$

(These profit functions are well-defined for all $\mathbf{p}_{c} \geq \mathbf{0}_{c}$, since $\mathbf{K}^{f}$ is compact for all $f$.)

The monopoly's strategy space $\mathbf{S}$ is $\Re^{(m+1)(H+F-1)+c}$. A strategy $\mathbf{s} \in \mathbf{S}$ consists of an offer to each household $h$ to sell $\mathbf{x}_{m}^{h}$ for charge $R^{h}$, an offer to each firm $f \neq m$ to sell $-\mathbf{y}_{m}^{f}$ for $R^{f}$, and finally the monopoly's competitive goods plan $\mathbf{y}_{c}^{m}$. Given household incomes $I^{h} \geq 0$ and competitive prices $\mathbf{p}_{c} \gg \mathbf{0}_{c}$, we say an offer $\left(\left(\mathbf{x}_{m}^{h}, R^{h}\right),\left(\mathbf{y}_{m}^{f}, R^{f}\right)_{f \neq m}\right)$ is acceptable if, for each household $h$ and competitive firm $f, \mathbf{x}_{m}^{h} \geq \mathbf{0}_{m}, \mathbf{y}_{m}^{f} \leq \mathbf{0}_{m}, R^{h} \leq I^{h}$, and the monopoly's offer is weakly beneficial, so that $V^{h}\left(\mathbf{x}_{m}^{h}, I^{h}-R^{h}, \mathbf{p}_{c}\right) \geq V^{h}\left(\mathbf{0}_{m}, I^{h}, \mathbf{p}_{c}\right)$ and $\Pi^{f}\left(\mathbf{y}_{m}^{f}, \mathbf{p}_{c}\right)-\Pi^{f}\left(\mathbf{0}_{m}, \mathbf{p}_{c}\right) \geq R^{f}$. A strategy can be produced if $\left(\sum_{h} \mathbf{x}_{m}^{h}-\sum_{f \neq m} \mathbf{y}_{m}^{f}, \mathbf{y}_{c}^{m}\right) \in \mathbf{Y}^{m}$. The monopoly solves [M]

$$
\max _{\left(\left(\mathbf{x}_{m}^{h}, R^{h}\right),\left(\mathbf{y}_{m}^{f}, R^{f}\right), \mathbf{y}_{c}^{m}\right)} \sum_{h} R^{h}+\sum_{f \neq m} R^{f}+\mathbf{p}_{c} \cdot \mathbf{y}_{c}^{m} \quad \text { subject to }
$$

(a) the offer $\left(\left(\mathbf{x}_{m}^{h}, R^{h}\right),\left(\mathbf{y}_{m}^{f}, R^{f}\right)_{f \neq m}\right)$ is acceptable; and

(b) the strategy $\left(\left(\mathbf{x}_{m}^{h}, R^{h}\right),\left(\mathbf{y}_{m}^{f}, R^{f}\right)_{f \neq m}, \mathbf{y}_{c}^{m}\right)$ can be produced. $[\mathbf{M}]$.

Naturally, the charges $R^{h}, R^{f}$ are all nonnegative for any solution to problem

\section{DEFINITION AND PROPERTIES OF EQUILIBRIUM}

An equilibrium in $E$ consists of a list of charges for the monopoly goods, consumption and production plans, and competitive goods prices such that markets clear and the monopoly, households, and competitive firms are all optimizing. More formally, we have the following definition. 
Definition 1: $\left\langle\left(R^{h *}\right),\left(R^{f *}\right)_{f \neq m},\left(\mathbf{x}^{h *}\right),\left(\mathbf{y}^{f *}\right), \mathbf{p}_{c}^{*}\right\rangle \in \Re^{H+F-1} \times \Re^{(m+c)(H+F)}$ $\times \Re^{c}$ is said to be a PDM equilibrium for the economy $E$ if given prices $\mathbf{p}_{c}^{*}$ and incomes $I^{h *} \equiv \mathbf{p}_{c}^{*} \cdot \boldsymbol{\omega}_{c}^{h}+\sum_{f \neq m} \theta^{h f}\left(\mathbf{p}_{c}^{*} \cdot \mathbf{y}_{c}^{f *}-R^{f *}\right)+\theta^{h m}\left(\sum_{\tilde{h}} R^{\tilde{h} *}+\sum_{f \neq m} R^{f *}+\right.$ $\left.\mathbf{p}_{c}^{*} \cdot \mathbf{y}_{c}^{m *}\right) \forall h$

1. $\left(\left(\mathbf{x}_{m}^{h *}, R^{h *}\right),\left(\mathbf{y}_{m}^{f *}, R^{f *}\right)_{f \neq m}, \mathbf{y}_{c}^{m *}\right)$ solves [M];

2. $U^{h}\left(\mathbf{x}^{h *}\right)=V^{h}\left(\mathbf{x}_{m}^{h *}, I^{h *}-R^{h *}, \mathbf{p}_{c}^{*}\right) \geq V^{h}\left(\mathbf{0}_{m}, I^{h *}, \mathbf{p}_{c}^{*}\right) \forall h$;

3. $\mathbf{p}_{c}^{*} \cdot \mathbf{y}_{c}^{f *}=\Pi^{f}\left(\mathbf{y}_{m}^{f *}, \mathbf{p}_{c}^{*}\right)$ and $\mathbf{p}_{c}^{*} \cdot \mathbf{y}_{c}^{f *}-R^{f *} \geq \Pi^{f}\left(\mathbf{0}_{m}, \mathbf{p}_{c}^{*}\right), \forall f \neq m$; and

4. $\mathbf{x}^{*}=\boldsymbol{\omega}+\mathbf{y}^{*}$.

Just as in an Arrow-Debreu economy, the monopoly takes household incomes and competitive prices as constant instead of doing general equilibrium forecasting. This behavioral assumption is reasonable for many firms, and even when it is not entirely appropriate, the assumption seems a sensible step for examining the robustness of partial equilibrium conclusions about profit maximization with price discrimination. Our monopoly's myopia distinguishes our equilibrium concept from those of Gabszewicz and Vial (1972) and the many subsequent general equilibrium papers which assume that imperfect competitors anticipate all price changes. These models engender controversy since it's not clear how a firm should compare profits when relative prices differ. The arbitrariness of such comparisons leads some authors to assert that any interior real allocation can be an equilibrium for some comparison rule (see Böhm (1990, p. 2)). It is worth noting that in our model, the prices of competitive goods pin down the meaning of profits and the equilibrium is independent of the normalization rule in that if $\left\langle\left(R^{h *}\right),\left(R^{f *}\right)_{f \neq m},\left(\mathbf{x}^{h *}\right),\left(\mathbf{y}^{f *}\right), \mathbf{p}_{c}^{*}\right\rangle$ is a PDM equilibrium, then so is $\left\langle\left(\alpha R^{h *}\right)\right.$, $\left.\left(\alpha R^{f *}\right)_{f \neq m},\left(\mathbf{x}^{h *}\right),\left(\mathbf{y}^{f *}\right), \alpha \mathbf{p}_{c}^{*}\right\rangle \forall \alpha>0$.

\subsection{An Inefficient Equilibrium}

Figure 3.1, Panel A, depicts a simple example of an inefficient PDM equilibrium. The two-good economy has one household with endowment $\boldsymbol{\omega}=(0,1)$ and utility $U\left(x_{1}, x_{2}\right)=x_{1}+x_{2}$, a monopoly with production set $\mathbf{Y}=\{(0,0),(2,-1)\}-$ $\Re_{+}^{2}$, and no competitive firms.

We claim that $\left\langle R=1, \mathbf{x}=(1,0), \mathbf{y}=(1,-1), p_{c}=1\right\rangle$ is a PDM equilibrium. In this equilibrium, monopoly profits are $\Pi=R+p_{c} y_{c}=1-1=0$ and household income is $I=p_{c} \omega_{c}+\Pi=1$. The monopoly cannot increase profits because even though it could costlessly increase production, the household can't afford to pay more, and the monopoly takes the household's income as given.

The reader can check that the unique Pareto optimum $\mathbf{x}=(2,0), \mathbf{y}=(2,-1)$ is part of another equilibrium with profits of 1 depicted in Figure 3.1, Panel B. As profits fall from 1 to 0 , the reservation utility level $V\left(0_{m}, \omega_{c}+\Pi\right)$ falls so that the example admits equilibria with consumption plans ranging from $(2,0)$ to $(1,0)$. This continuum of equilibria may remind readers of the indeterminacy of equilibria in previous general equilibrium models of imperfect competition (see Böhm (1994) and Grodal (1984)). However, the next section shows that PDM equilibria are typically efficient. 
Panel A

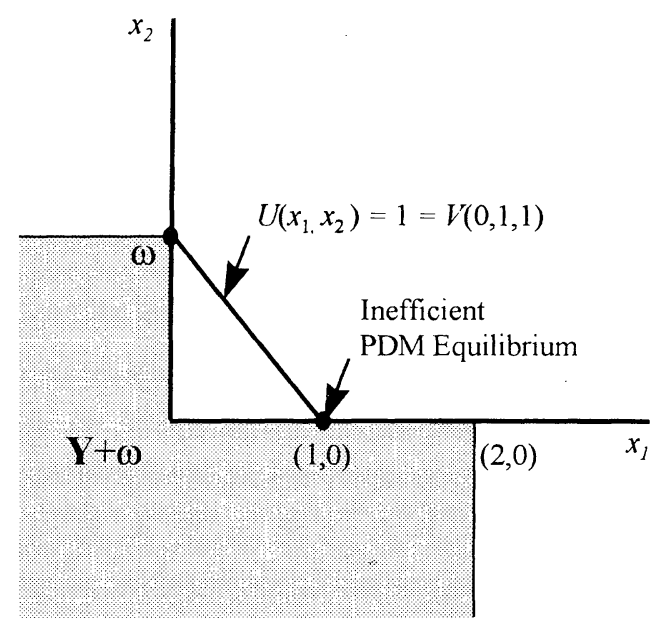

Panel B

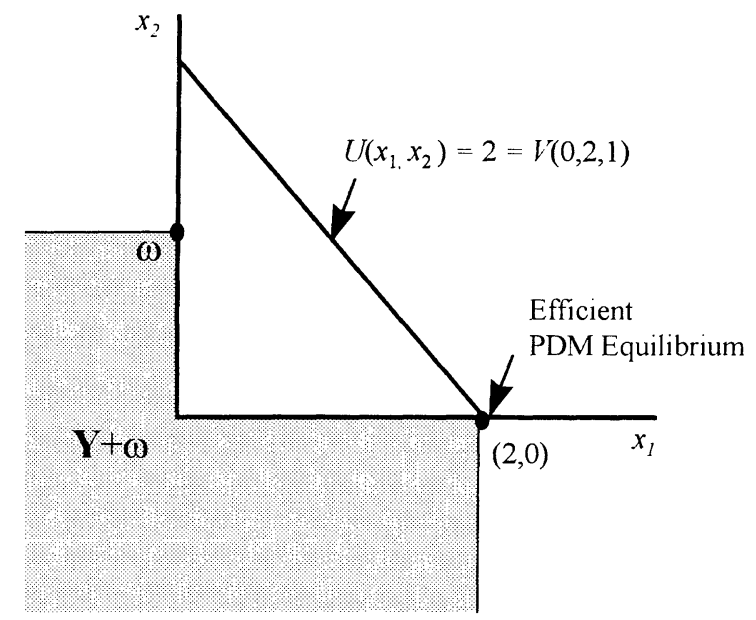

FIGURE 3.1.-Inefficient and efficient equilibria.

\subsection{Efficient Equilibria}

Condition (F-1) below precludes technologies that admit inefficient PDM equilibria. It asserts that the monopoly can reduce its input or increase its output of competitive goods whenever it reduces its output of monopoly goods.

(F-1) $\forall \mathbf{y}^{m} \in \mathbf{Y}^{m}$ where $\mathbf{y}_{m}^{m}>\mathbf{0}_{m}$, and $\forall \mathbf{z}_{m}$ where $\mathbf{0}_{m} \leq \mathbf{y}_{m}^{m}-\mathbf{z}_{m}<\mathbf{y}_{m}^{m}, \exists \mathbf{z}_{c}>\mathbf{0}_{c}$ such that $\mathbf{y}^{m}+\left(-\mathbf{z}_{m}, \mathbf{z}_{c}\right) \in \mathbf{Y}^{m}$.

This condition says that the monopoly goods are costly in that discrete reductions in output imply discrete reductions in cost. It eliminates the technol- 
ogy in Figure 3.1 together with several found elsewhere in the increasing returns literature (see, e.g., Brown and Heal (1979)), but allows some technologies that have unbounded marginal returns in Vohra's (1992) terminology, since the monopoly goods could be costless at the margin. (We will subsequently introduce (F-1)', a stronger assumption that eliminates such technologies as well.)

\section{THEOREM 3.1 (First Welfare Theorem): PDM equilibria are Pareto efficient if} ( F-1) holds.

Proof: Let (F-1) hold and $\left\langle\left(R^{h *}\right),\left(R^{f *}\right)_{f \neq m},\left(\mathbf{x}^{h *}\right),\left(\mathbf{y}^{f *}\right), \mathbf{p}_{c}^{*}\right\rangle$ be an equilibrium. We show that the allocation $\left(\mathbf{x}^{h *}\right),\left(\mathbf{y}^{f *}\right)$ must be efficient by arguing that any Pareto superior allocation $\left(\hat{\mathbf{x}}^{h}\right),\left(\hat{\mathbf{y}}^{f}\right)$ must be infeasible, because if it were feasible, then the monopoly could make higher profits than in the equilibrium.

Step 1: If $\left(\hat{\mathbf{x}}^{h}\right),\left(\hat{\mathbf{y}}^{f}\right)$ were feasible, the strategy $\hat{\mathbf{s}} \equiv\left(\left(\hat{\mathbf{x}}_{m}^{h}, \hat{R}^{h}\right),\left(\hat{\mathbf{y}}_{m}^{f}, \hat{R}^{f}\right)_{f \neq m}, \hat{\mathbf{y}}_{c}^{m}\right)$, where $\hat{R}^{h} \equiv R^{h *}+\mathbf{p}_{c}^{*} \cdot\left(\mathbf{x}_{c}^{h *}-\hat{\mathbf{x}}_{c}^{h}\right)$ and $\hat{R}^{f} \equiv R^{f *}+\mathbf{p}_{c}^{*} \cdot\left(\hat{\mathbf{y}}_{c}^{f}-\mathbf{y}_{c}^{f *}\right)$, would be at least as profitable as the equilibrium strategy.

The offer $\left(\hat{\mathbf{x}}_{m}^{h}, \hat{R}^{h}\right)$ is acceptable to each household $h$, because $\hat{\mathbf{x}}^{h}$ is affordable (it costs the same as $\mathbf{x}^{h *}$ did) and yields at least the utility of $\mathbf{x}^{h *}$, which must in turn yield at least the reservation utility since $\mathbf{x}^{h *}$ was chosen in equilibrium. The offer is acceptable to each competitive firm $f$ because

$$
\Pi^{f}\left(\hat{\mathbf{y}}_{m}^{f}, \mathbf{p}_{c}^{*}\right) \geq \mathbf{p}_{c}^{*} \cdot \hat{\mathbf{y}}_{c}^{f}=\hat{R}^{f}+\mathbf{p}_{c}^{*} \cdot \mathbf{y}_{c}^{f *}-R^{f *} \geq \hat{R}^{f}+\Pi^{f}\left(\mathbf{0}_{m}, \mathbf{p}_{c}^{*}\right) .
$$

(The first inequality holds because feasibility implies $\left(\hat{\mathbf{y}}_{m}^{f}, \hat{\mathbf{y}}_{c}^{f}\right) \in \mathbf{Y}^{f}$, the equality because of the construction of $\hat{R}^{f}$, and the second inequality because the equilibrium offer was acceptable.)

Monopoly profits $\hat{\Pi}^{m}$ from strategy $\hat{\mathbf{s}}$ equal

$$
\sum_{h}\left[R^{h *}+\mathbf{p}_{c}^{*} \cdot\left(\mathbf{x}_{c}^{h *}-\hat{\mathbf{x}}_{c}^{h}\right)\right]+\sum_{f \neq m}\left[R^{f *}+\mathbf{p}_{c}^{*} \cdot\left(\hat{\mathbf{y}}_{c}^{f}-\mathbf{y}_{c}^{f *}\right)\right]+\mathbf{p}_{c}^{*} \cdot \hat{\mathbf{y}}_{c}^{m},
$$

whereas equilibrium profits $\Pi^{m *}$ equal $\sum_{h} R^{h *}+\sum_{f \neq m} R^{f *}+\mathbf{p}_{c}^{*} \cdot \mathbf{y}_{c}^{m *}$. The difference is $\hat{\Pi}^{m}-\Pi^{m *}=\mathbf{p}_{c}^{*} \cdot\left[\left(\mathbf{x}_{c}^{*}-\mathbf{y}_{c}^{*}\right)-\left(\hat{\mathbf{x}}_{c}-\hat{\mathbf{y}}_{c}\right)\right]=\mathbf{p}_{c}^{*} \cdot\left[\boldsymbol{\omega}_{c}-\left(\hat{\mathbf{x}}_{c}-\hat{\mathbf{y}}_{c}\right)\right]$. If the allocation $\left(\hat{\mathbf{x}}^{h}\right),\left(\hat{\mathbf{y}}^{f}\right)$ were feasible, then $\boldsymbol{\omega}_{c} \geq \hat{\mathbf{x}}_{c}-\hat{\mathbf{y}}_{c}$, which would imply that $\hat{\Pi}^{m} \geq \Pi^{m *}$.

Step 2: The monopoly could strictly increase profits from $\hat{\mathbf{s}}$ by offering less of the monopoly goods or increasing the charge to some household.

Let $h^{\prime}$ be a household strictly better off.

Case $A$ : $\hat{\mathbf{x}}_{c}^{h^{\prime}}>\mathbf{0}_{c}$. Then, for sufficiently small $\varepsilon$, the monopoly can make more profit by offering $\left(\hat{\mathbf{x}}_{m}^{h^{\prime}}, \hat{R}^{h^{\prime}}+\varepsilon\right)$ and otherwise leaving $\hat{\mathbf{s}}$ unchanged.

Case $B: \hat{\mathbf{x}}_{c}^{h^{\prime}}=\mathbf{0}_{c}$. Then, $\hat{\mathbf{x}}_{m}^{h^{\prime}}>\mathbf{0}_{m}$ since $U^{h^{\prime}}\left(\hat{\mathbf{x}}^{h^{\prime}}\right)>U^{h^{\prime}}\left(\mathbf{x}^{h^{\prime} *}\right)$. The monopoly cannot increase its charge since the household is already spending its entire 
income on the monopoly goods. The monopoly can, however, take advantage of the household's strict preference for $\hat{\mathbf{x}}^{h^{\prime}}$ by decreasing sales to $h^{\prime}$. Condition (F-1) implies that for all $\boldsymbol{\varepsilon}_{m}$ such that $\hat{\mathbf{y}}_{m}^{m}>\boldsymbol{\varepsilon}_{m}>\mathbf{0}_{m}, \exists \boldsymbol{\varepsilon}_{c}>\mathbf{0}_{c}$ such that $\hat{\mathbf{y}}^{m}+\left(-\boldsymbol{\varepsilon}_{m}, \boldsymbol{\varepsilon}_{c}\right) \in \mathbf{Y}^{m}$. For sufficiently small $\boldsymbol{\varepsilon}_{m}>\boldsymbol{0}_{m}$, the monopoly can make profits $\hat{\Pi}^{m}+\mathbf{p}_{c}^{*} \cdot \boldsymbol{\varepsilon}_{c}>\hat{\Pi}^{m}$ by offering $\left(\hat{\mathbf{x}}_{m}^{h^{\prime}}-\boldsymbol{\varepsilon}_{m}, \hat{R}^{h^{\prime}}\right)$ to $h^{\prime}$, adopting the competitive goods plan $\hat{\mathbf{y}}_{c}^{m}+\boldsymbol{\varepsilon}_{c}$ and otherwise leaving $\hat{\mathbf{s}}$ unchanged. ${ }^{5}$

Q.E.D.

This theorem validates the partial equilibrium conclusion that perfect price discrimination is generally efficient: PDM equilibria are only inefficient if the monopoly cannot reduce cost by cutting output. Our efficiency argument differs from the standard competitive argument in Arrow (1951) because in our model the set of preferred bundles is not separated from the production possibility set by a hyperplane. As we will see in Section 4.1, these sets are separated by the monopoly's isoprofit set, a nonlinear hypersurface.

\subsection{Failures of Decentralization and Existence}

Vohra (1990, pp. 429-432), Quinzii (1992, pp. 36-42), and Brown, Heller, and Starr (1992, pp. 67-69) all provide examples of economies with increasing returns for which Pareto optima cannot be decentralized as two-part tariff equilibria. In each case, there is too little willingness to pay to run the regulated monopoly, so these optima also cannot be decentralized as PDM equilibria., In fact, no PDM equilibrium exists in these economies.

One might conjecture that the existence and decentralization problems of our equilibrium notion would disappear in economies with sufficient willingness-topay, as they do for the two-part tariff equilibria of Brown, Heller, and Starr (1992). However, the following example has a unique Pareto optimum which cannot be decentralized as a PDM equilibrium, not because it has inadequate surplus, but because some other production plan, one with even more surplus, attracts the monopoly. The example also shows that equilibria may not exist, though we leave it to the reader to check nonexistence for this case. ${ }^{8,9}$

\footnotetext{
${ }^{5}$ Note that $\mathbf{p}_{c}^{*} \gg \mathbf{0}_{c}$ since preferences are strictly monotonic.

${ }^{6}$ In Vohra's example, firm 2 (the natural monopoly) produces a perfect substitute for the good of firm 1. Firm 2's average cost lies above firm 1's marginal and average cost when firm 1 produces little or nothing, but below firm 1's marginal and average cost when firm 1 produces a lot. Consequently, if only firm 2 produces, it loses money since it must price below the price of firm 1, and if only firm 1 produces, the price for (and cost of) firm 1's goods rise enough to attract firm 2's entry. Even though it may be efficient for firm 2 to produce alone, there is insufficient willingness to pay because of the competition offered by firm 1's good.

${ }^{7}$ These authors restrict attention to individualized two-part tariffs, but Theorem 4.1 shows that if the monopoly cannot cover its costs using such tariffs, it cannot do so with any pricing scheme.

${ }^{8}$ If (F-1) held, then nonexistence would follow from the facts that: (i) the unique Pareto optimum cannot be decentralized, and (ii) all PDM equilibria are efficient when (F-1) holds.

${ }^{9}$ Our model does not address what happens when some agent tries to maximize surplus and no equilibrium exists, but one can easily imagine that this would lead to inefficiencies and a sort of refutation of the notion that perfect price discrimination is efficient.
} 
Consider an economy with three goods, one household with endowment $\boldsymbol{\omega}=(0,9,9)$ and utility $U\left(x_{1}, x_{2}, x_{3}\right)=x_{2}^{1 / 2} x_{3}^{1 / 2}+4 x_{1}$, a monopoly with production set $\mathbf{Y}=\{(0,0,0),(1,-5,0),(2,-7.01,-7)\}-\mathfrak{R}_{+}^{3}$, and no competitive firms. Note that $U(1,4,9)=10>U(2,1.99,2)=9.99>U(0,9,9)=9$. Hence, the unique Pareto optimum is $\mathbf{y}=(1,-5,0), \mathbf{x}=(1,4,9)$.

Supporting the consumption $(1,4,9)$ in a decentralization of the optimum requires that $p_{2} / p_{3}=9 / 4$, so w.l.o.g. let $\mathbf{p}_{c}=(9,4)$. Because the PDM will extract the full surplus in equilibrium, the household must have income $I$ such that $U(1,4,9)=V\left(0, I, \mathbf{p}_{c}\right)$. Hence, $I$ equals $120=\min \mathbf{p}_{c} \cdot \mathbf{x}_{c}$ subject to $U\left(0, \mathbf{x}_{c}\right)$ $=10$. Finally, the monopoly charge must be $R=I-\mathbf{p}_{c} \cdot \mathbf{x}_{c}=120-(9,4) \cdot(4,9)$ $=48$, yielding profits of $48+\mathbf{p}_{c} \cdot \mathbf{y}_{c}=3$. The reader can check that $\langle\mathbf{x}=(1,4,9)$, $\left.R=48, \mathbf{y}=(1,-5,0), \mathbf{p}_{c}=(9,4)\right\rangle$ satisfies equilibrium conditions $2-4$. However, it does not satisfy condition 1 , since the offer $\left(x_{m}=2, R=96\right)$ is acceptable ${ }^{10}$ and the strategy $\left\langle\left(x_{m}=2, R=96\right), \mathbf{y}_{c}=(-7.01,-7)\right\rangle$. can be produced, and yields profits of $4.91=96-(9 \times 7.01+4 \times 7)>3$. Consequently, the Pareto optimum cannot be decentralized as a PDM equilibrium. The monopoly wants to produce the extra unit of output because the household's willingness to pay is high and the cost of producing the extra unit is low, lower in fact than would be possible if the technology were convex. ${ }^{11}$

\section{ISO-PROFIT SETS AND TWO-PART TARIFFS}

This section explores more thoroughly what it means for a PDM to maximize profits. In particular, we characterize a PDM's iso-profit sets together with the set of production plans that yield higher profits than in some candidate equilibrium. This analysis provides a geometric understanding of the results in the previous section. It also helps us generalize the finding in the partial equilibrium literature (see, e.g., Oi (1971)) that a monopoly can perfectly discriminate by charging individualized hookup fees plus a uniform per-unit price equal to marginal cost.

\subsection{Iso-Profit Sets}

In a competitive economy, an iso-profit set consists of production plans yielding identical profits at given prices $\mathbf{p}$, i.e., $\{\mathbf{y} \mid \mathbf{p} \cdot \mathbf{y}=$ constant $\}$. In contrast, a PDM's iso-profit set depends on both the competitive prices and household incomes. Given prices $\mathbf{p}_{c} \gg \mathbf{0}_{c}$ and incomes $\left(I^{h}\right)$, it can be written as

$$
\left\{\mathbf{y}^{m} \in \Re^{m+c} \mid \Pi^{m}\left(\mathbf{y}^{m}, \mathbf{p}_{c},\left(I^{h}\right)\right)=\text { constant }\right\},
$$

\footnotetext{
${ }^{10}$ The offer $\left(x_{m}=2, R=96\right)$ is acceptable because $V\left(2, I-R, \mathbf{p}_{c}\right)=U\left(2, \frac{4}{3}, 3\right)=10=V\left(0, I, \mathbf{p}_{c}\right)$.

${ }^{11}$ In Section 5, we show that Pareto optima can be decentralized when production technologies are convex.
} 
where monopoly profits $\Pi^{m}(\cdot)$ and revenues $R(\cdot)$ are defined as follows:

$$
\begin{aligned}
& \Pi^{m}\left(\mathbf{y}^{m}, \mathbf{p}_{c},\left(I^{h}\right)\right) \equiv R\left(\mathbf{y}_{m}^{m}, \mathbf{p}_{c},\left(I^{h}\right)\right)+\mathbf{p}_{c} \cdot \mathbf{y}_{c}^{m} \\
& R\left(\mathbf{y}_{m}^{m}, \mathbf{p}_{c},\left(I^{h}\right)\right) \equiv \max _{\left(\mathbf{x}_{m}^{h}, R^{h}\right),\left(\mathbf{y}_{m}^{f}, R^{f}\right)_{f \neq m}} \sum R^{h}+\sum_{f \neq m} R^{f} \quad \text { subject to } \\
& \text { (a) the offer }\left(\left(\mathbf{x}_{m}^{h}, R^{h}\right),\left(\mathbf{y}_{m}^{f}, R^{f}\right)_{f \neq m}\right) \text { is acceptable; } \\
& \text { (b) } \mathbf{y}_{m}^{m}=\sum_{h} \mathbf{x}_{m}^{h}-\sum_{f \neq m} \mathbf{y}_{m}^{f} .
\end{aligned}
$$

We require a few more definitions in order to characterize the revenue function $R(\cdot)$ in Lemma 1. Define weakly-preferred sets as

$$
\mathbf{B}^{h}\left(\mathbf{p}_{c}, I^{h}\right) \equiv\left\{\mathbf{x} \in \Re_{+}^{m+c} \mid U^{h}(\mathbf{x}) \geq V^{h}\left(\mathbf{0}_{m}, I^{h}, \mathbf{p}_{c}\right)\right\} \quad \forall h,
$$

and let

$$
\begin{aligned}
& \mathbf{H}\left(\mathbf{p}_{c}\right) \equiv\left\{\left(\mathbf{0}_{m}, \mathbf{x}_{c}\right) \in \mathfrak{R}^{m+c} \mid \mathbf{p}_{c} \cdot \mathbf{x}_{c}=0\right\}, \\
& \mathbf{H}^{+}\left(\mathbf{p}_{c}\right) \equiv\left\{\left(\mathbf{0}_{m}, \mathbf{x}_{c}\right) \in \Re^{m+c} \mid \mathbf{p}_{c} \cdot \mathbf{x}_{c}>0\right\}, \\
& \mathbf{Z}\left(\mathbf{p}_{c},\left(I^{h}\right)\right) \equiv \sum_{h} \mathbf{B}^{h}\left(\mathbf{p}_{c}, I^{h}\right)-\sum_{f \neq m} \mathbf{Y}^{f}+\mathbf{H}\left(\mathbf{p}_{c}\right), \\
& \mathbf{Z}^{+}\left(\mathbf{p}_{c},\left(I^{h}\right)\right) \equiv \sum_{h} \mathbf{B}^{h}\left(\mathbf{p}_{c}, I^{h}\right)-\sum_{f \neq m} \mathbf{Y}^{f}+\mathbf{H}^{+}\left(\mathbf{p}_{c}\right), \quad \text { and } \\
& \Gamma\left(\mathbf{y}_{m}, \mathbf{p}_{c},\left(I^{h}\right)\right) \equiv \min _{\mathbf{x} \in \Re^{c}} \mathbf{p}_{c} \cdot \mathbf{x} \quad \text { subject to } \quad\left(\mathbf{y}_{m}, \mathbf{x}\right) \in \mathbf{Z}\left(\mathbf{p}_{c},\left(I^{h}\right)\right) .
\end{aligned}
$$

Observe that $\mathbf{Z}(\cdot) \supseteq \sum \mathbf{B}^{h}(\cdot)-\sum_{f \neq m} \mathbf{Y}^{f}$ (with equality holding if $c=1$ ). If we restrict these sets to some subspace where the monopoly goods are held constant, then the boundary of $\mathbf{Z}(\cdot)$, denoted $\partial \mathbf{Z}(\cdot)$, is a $(c-1)$ dimensional hyperplane supporting $\sum \mathbf{B}^{h}(\cdot)-\sum_{f \neq m} \mathbf{Y}^{f}$ with expenditures $\Gamma(\cdot)$ on competitive goods. (Figure 4.1 depicts two such hyperplanes.) A stack of such hyperplanes forms $\partial \mathbf{Z}(\cdot)$, a nonlinear hypersurface that follows the boundary of $\sum \mathbf{B}^{h}(\cdot)-$ $\sum_{f \neq m} \mathbf{Y}^{f}$, supporting it for each level of monopoly goods. Revenues are determined by minimizing expenditure on this set, as shown below.

LEMMA 1: $R\left(\mathbf{y}_{m}, \mathbf{p}_{c},\left(I^{h}\right)\right)=\sum I^{h}-\Gamma\left(\mathbf{y}_{m}, \mathbf{p}_{c},\left(I^{h}\right)\right)-\sum_{f \neq m} \Pi^{f}\left(\mathbf{0}_{m}, \mathbf{p}_{c}\right), \quad \forall \mathbf{p}_{c} \gg$ $\mathbf{0}_{c}, \mathbf{y}_{m} \geq \mathbf{0}_{m},\left(I^{h} \geq 0\right)$.

Proof: Consider any $\mathbf{p}_{c} \gg \mathbf{0}_{c}, \mathbf{y}_{m} \geq \mathbf{0}_{m},\left(I^{h} \geq 0\right)$. The construction of $\mathbf{Z}(\cdot)$ and $\Gamma(\cdot)$ implies that there exist $\left(\hat{\mathbf{x}}^{h} \in \mathbf{B}^{h}\left(\mathbf{p}_{c}, I^{h}\right)\right),\left(\hat{\mathbf{y}}^{f} \in \mathbf{Y}^{f}\right)_{f \neq m}, \hat{\mathbf{h}} \in \mathbf{H}\left(\mathbf{p}_{c}\right)$, such that

$$
\Gamma\left(\mathbf{y}_{m}, \mathbf{p}_{c},\left(I^{h}\right)\right)=\mathbf{p}_{c} \cdot\left(\sum_{h} \hat{\mathbf{x}}_{c}^{h}-\sum_{f \neq m} \hat{\mathbf{y}}_{c}^{f}+\hat{\mathbf{h}}_{c}\right)=\mathbf{p}_{c} \cdot\left(\sum_{h} \hat{\mathbf{x}}_{c}^{h}-\sum_{f \neq m} \hat{\mathbf{y}}_{c}^{f}\right),
$$

and

$$
\mathbf{y}_{m}=\sum_{h} \hat{\mathbf{x}}_{m}^{h}-\sum_{f \neq m} \hat{\mathbf{y}}_{m}^{f}+\hat{\mathbf{h}}_{m}=\sum_{h} \hat{\mathbf{x}}_{m}^{h}-\sum_{f \neq m} \hat{\mathbf{y}}_{m}^{f}
$$


Panel A

Good m+1

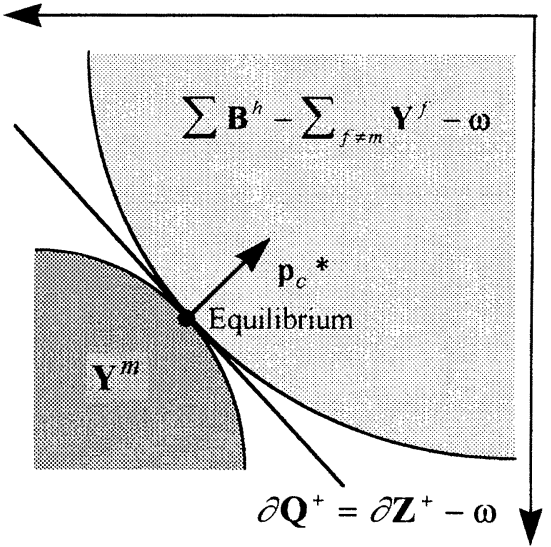

Good $\mathrm{m}+2$

Panel B

Good m+1

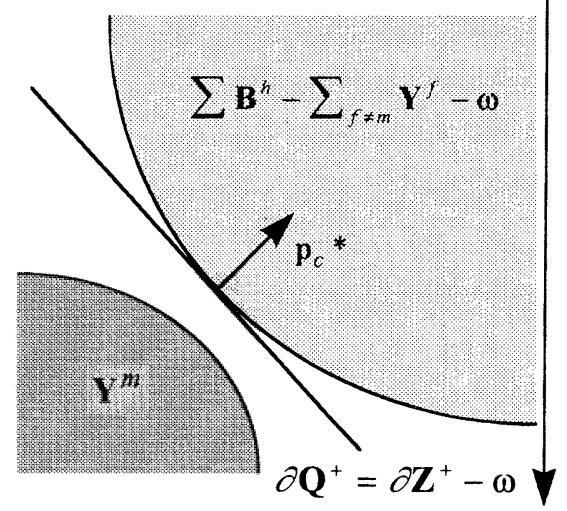

Good $\mathrm{m}+2$

Figure 4.1.- The boundary of $\mathbf{Q}^{+}$separates $\Sigma \mathbf{B}^{h}-\sum_{f \neq m} \mathbf{Y}^{f}-\boldsymbol{\omega}$ from $\mathbf{Y}^{m}$ in an efficient equilibrium. The cross-section in Panel A holds the $m$ monopoly goods equal to their equilibrium level $\mathbf{y}_{m}^{m *}$; Panel B depicts another cross-section. 
Since $\hat{\mathbf{x}}^{h} \in \mathbf{B}^{h}\left(\mathbf{p}_{c}, I^{h}\right) \forall h$ and $\hat{\mathbf{y}}^{f} \in \mathbf{Y}^{f} \forall f \neq m$, the offer

$$
\left\langle\left(\hat{\mathbf{x}}_{m}^{h}, \hat{R}^{h} \equiv I^{h}-\mathbf{p}_{c} \cdot \hat{\mathbf{x}}_{c}^{h}\right),\left(\hat{\mathbf{y}}_{m}^{f}, \hat{R}^{f} \equiv \mathbf{p}_{c} \cdot \hat{\mathbf{y}}_{c}^{f}-\Pi^{f}\left(\mathbf{0}_{m}, \mathbf{p}_{c}\right)\right)_{f \neq m}\right\rangle
$$

is acceptable. Hence,

$$
\begin{aligned}
R\left(\mathbf{y}_{m}, \mathbf{p}_{c},\left(I^{h}\right)\right) & \geq \sum I^{h}-\mathbf{p}_{c} \cdot \sum_{h} \hat{\mathbf{x}}_{c}^{h}+\mathbf{p}_{c} \cdot \sum_{f \neq m} \hat{\mathbf{y}}_{c}^{f}-\sum_{f \neq m} \Pi^{f}\left(\mathbf{0}_{m}, \mathbf{p}_{c}\right) \\
& =\sum I^{h}-\Gamma\left(\mathbf{y}_{m}, \mathbf{p}_{c},\left(I^{h}\right)\right)-\sum_{f \neq m} \Pi^{f}\left(\mathbf{0}_{m}, \mathbf{p}_{c}\right) .
\end{aligned}
$$

It remains only to show that the revenues from any acceptable offer are never strictly larger than the right-hand side in the above inequality. If $\left(\left(\tilde{\mathbf{x}}_{m}^{h}, \tilde{R}^{h}\right),\left(\tilde{\mathbf{y}}_{m}^{f}\right.\right.$, $\left.\tilde{R}^{f}\right)_{f \neq m}$ ) is an acceptable offer for which $\mathbf{y}_{m}=\sum_{\mathbf{x}_{m}^{h}}^{h}-\sum_{f \neq m} \tilde{\mathbf{y}}_{m}^{f}$, then there exist $\left(\tilde{\mathbf{x}}_{c}^{h}\right),\left(\tilde{\mathbf{y}}_{c}^{f}\right)_{f \neq m}$ such that $\left(\tilde{\mathbf{x}}_{m}^{h}, \tilde{\mathbf{x}}_{c}^{h}\right) \in \mathbf{B}^{h}\left(\mathbf{p}_{c}, I^{h}\right),\left(\tilde{\mathbf{y}}_{m}^{f}, \tilde{\mathbf{y}}_{c}^{f}\right) \in \mathbf{Y}^{f}, \tilde{R}^{h} \leq I^{h}-\mathbf{p}_{c} \cdot \tilde{\mathbf{x}}_{c}^{h}$, and $\tilde{R}^{f} \leq \mathbf{p}_{c} \cdot \tilde{\mathbf{y}}_{c}^{f}-\Pi^{f}\left(\mathbf{0}_{m}, \mathbf{p}_{c}\right)$. Hence, the revenue from this offer is at most

$$
\sum_{h} I^{h}-\mathbf{p}_{c} \cdot \sum_{h} \tilde{\mathbf{x}}_{c}^{h}+\mathbf{p}_{c} \cdot \sum_{f \neq m} \tilde{\mathbf{y}}_{c}^{f}-\sum_{f \neq m} \Pi^{f}\left(\mathbf{0}_{m}, \mathbf{p}_{c}\right),
$$

which by the construction of $\Gamma(\cdot)$ is less than or equal to

$$
\sum_{h} I^{h}-\Gamma\left(\mathbf{y}_{m}, \mathbf{p}_{c},\left(I^{h}\right)\right)-\sum_{f \neq m} \Pi^{f}\left(\mathbf{0}_{m}, \mathbf{p}_{c}\right) .
$$

We next use Lemma 1 to characterize $\mathbf{Q}^{+}$, the set of production plans that would yield the monopoly higher profits than it gets in a "candidate equilibrium."

Definition 2: $\left\langle\left(R^{h *}\right),\left(R^{f *}\right)_{f \neq m},\left(\mathbf{x}^{h *}\right),\left(\mathbf{y}^{f *}\right), \mathbf{p}_{c}^{*}\right\rangle$ is said to be a candidate equilibrium if conditions (2), (3), and (4) from Definition 1 are satisfied given incomes $I^{h *} \equiv R^{h *}+\mathbf{p}_{c}^{*} \cdot \mathbf{x}_{c}^{h *} \forall h$, and if $\mathbf{p}_{c}^{*} \cdot \mathbf{y}_{c}^{f *}-R^{f *}=\Pi^{f}\left(\mathbf{0}_{m}, \mathbf{p}_{c}^{*}\right) \forall f \neq m$.

A candidate equilibrium differs from an equilibrium in two ways. First, the monopoly problem may not be solved, and second, the candidate equilibrium may implicitly involve redistribution, since $R^{h *}+\mathbf{p}_{c}^{*} \cdot \mathbf{x}_{c}^{h *}$ could differ from $\mathbf{p}_{c}^{*} \cdot \boldsymbol{\omega}_{c}^{h}+\sum_{f \neq m} \theta^{h f}\left(\mathbf{p}_{c}^{*} \cdot \mathbf{y}_{c}^{f *}-R^{f *}\right)+\theta^{h m}\left(\sum_{\tilde{h}} R^{\tilde{h} *}+\sum_{f \neq m} R^{f *}+\mathbf{p}_{c}^{*} \cdot \mathbf{y}_{c}^{m *}\right)$.

LEMMA 2: Let $\left\langle\left(R^{h *}\right),\left(R^{f *}\right)_{f \neq m},\left(\mathbf{x}^{h *}\right),\left(\mathbf{y}^{f *}\right), \mathbf{p}_{c}^{*}\right\rangle$ be a candidate equilibrium and let $\mathbf{Q}^{+} \equiv\left\{\mathbf{y} \in \Re^{m+c} \mid \Pi^{m}\left(\mathbf{y}, \mathbf{p}_{c}^{*},\left(I^{h *}\right)\right)>\sum R^{h *}+\sum_{f \neq m} R^{f *}+\mathbf{p}_{c}^{*} \cdot \mathbf{y}_{c}^{m *}\right\}$, where $I^{h *}=R^{h *}+\mathbf{p}_{c}^{*} \cdot \mathbf{x}_{c}^{h *}$. Then $\mathbf{Q}^{+}=\mathbf{Z}^{+}\left(\mathbf{p}_{c}^{*},\left(I^{h *}\right)\right)-\boldsymbol{\omega}$.

Proof: $\mathbf{y} \in \mathbf{Q}^{+} \Leftrightarrow$

$$
\begin{aligned}
\mathbf{p}_{c}^{*} \cdot \mathbf{y}_{c} & +R\left(\mathbf{y}_{m}, \mathbf{p}_{c}^{*},\left(I^{h *}\right)\right) \\
& >\mathbf{p}_{c}^{*} \cdot \mathbf{y}_{c}^{m *}+\sum_{h}\left(I^{h *}-\mathbf{p}_{c}^{*} \cdot \mathbf{x}_{c}^{h *}\right)+\sum_{f \neq m}\left[\mathbf{p}_{c}^{*} \cdot \mathbf{y}_{c}^{f *}-\Pi^{f}\left(\mathbf{0}_{m}, \mathbf{p}_{c}^{*}\right)\right] \\
& =\sum_{h} I^{h *}-\sum_{f \neq m} \Pi^{f}\left(\mathbf{0}_{m}, \mathbf{p}_{c}^{*}\right)-\mathbf{p}_{c}^{*} \cdot \boldsymbol{\omega}_{c} .
\end{aligned}
$$


Using Lemma 1 to substitute for $R\left(\mathbf{y}_{m}, \mathbf{p}_{c}^{*},\left(I^{h *}\right)\right)$ yields

$$
\mathbf{p}_{c}^{*} \cdot \mathbf{y}_{c}>\Gamma\left(\mathbf{y}_{m}, \mathbf{p}_{c}^{*},\left(I^{h *}\right)\right)-\mathbf{p}_{c}^{*} \cdot \boldsymbol{\omega}_{c},
$$

which is equivalent to $\mathbf{y} \in \mathbf{Z}^{+}\left(\mathbf{p}_{c}^{*},\left(I^{h *}\right)\right)-\boldsymbol{\omega}^{12}$

Lemma 2 helps us to interpret the results in Section 3. In an equilibrium, the PDM cannot increase profits, so $\mathbf{Y}^{m} \cap \mathbf{Q}^{+}=\varnothing$. Except for boundary problems, this empty intersection implies that equilibria are efficient since $\mathbf{Q}^{+}$contains the interior of $\sum \mathbf{B}^{h}(\cdot)-\sum_{f \neq m} \mathbf{Y}^{f}-\boldsymbol{\omega}^{13}$ The boundary of $\mathbf{Q}^{+}$is a nonlinear hypersurface separating the interior of $\sum \mathbf{B}^{h}(\cdot)-\sum_{f \neq m} \mathbf{Y}^{f}-\boldsymbol{\omega}$ from $\mathbf{Y}^{m}$ just as a hyperplane separates these sets in a competitive equilibrium. Figure 4.1 depicts this separation in two cross sections, one holding the monopoly goods constant at the equilibrium level $\mathbf{y}_{m}^{m *}$ and another at $\mathbf{y}_{m}^{m \prime}$. The figure looks like the competitive case; however, if we could depict a third dimension representing the monopoly goods, then we would see that $\mathbf{Q}^{+}$curves to hug $\sum \mathbf{B}^{h}(\cdot)+\sum_{f \neq m} \mathbf{Y}^{f}-\boldsymbol{\omega}$, and unlike its competitive counterpart, is generally not a halfspace.

A Pareto equilibrium can be decentralized if the monopoly problem is solved at the candidate equilibrium associated with the Pareto optimum, i.e., if $\mathbf{Y}^{m} \cap$ $\mathbf{Q}^{+}=\varnothing$. However, when $c>1$, the boundary of $\mathbf{Q}^{+}$can differ from that of $\sum \mathbf{B}^{h}(\cdot)+\sum_{f \neq m} \mathbf{Y}^{f}-\boldsymbol{\omega}$, and hence $\mathbf{Y}^{m}$ can cut inside $\mathbf{Q}^{+}$without violating Pareto optimality. Such an intersection precludes decentralization. The problem might be simply that the monopoly is not minimizing cost at the production plan in the optimum. On the other hand, if the cross-sections, $\left\{\left(\mathbf{y}_{m}, \mathbf{y}_{c}\right) \in \mathbf{Y}^{m} \mid \mathbf{y}_{m}=\tilde{\mathbf{y}}_{m}\right\}$, are all convex, as in Figures 4.1 and 4.2, the nonconvexities in producing the monopoly goods must cause any decentralization problem. In this case, the intersection of $\mathbf{Y}^{m}$ and $\mathbf{Q}^{+}$must occur at some monopoly production level other than the optimum, as it did in the example in Section 3.3 depicted in Figure 4.2. Such an intersection requires that production nonconvexities be sufficiently large that they overwhelm the convexity that $\mathbf{Q}^{+}$inherits from the households' preferences and the competitive firms' technologies. To be more specific, recall that at a Pareto optimum, Kahn and Vohra's (1987) Generalized Second Welfare Theorem gives a price vector $\mathbf{p} \in \Re_{+}^{m+c}, \mathbf{p} \neq 0$, which reflects marginal costs and marginal rates of substitution. This price reflects the marginal willingness of buyers and competitive firms to pay for the monopoly goods, given the

${ }^{12}$ If $\boldsymbol{\omega}+\mathbf{y} \in \mathbf{Z}^{+}\left(\mathbf{p}_{c}^{*},\left(I^{h *}\right)\right)$, then there exists $\left(\mathbf{0}_{m}, \mathbf{h}_{c}\right) \in \mathbf{H}^{+}\left(\mathbf{p}_{c}^{*}\right)$ such that $\boldsymbol{\omega}+\mathbf{y}-\left(\mathbf{0}_{m}, \mathbf{h}_{c}\right) \in$ $\mathbf{Z}\left(\mathbf{p}_{c}^{*},\left(I^{h *}\right)\right)$, which implies that $\mathbf{p}_{c}^{*} \cdot\left(\mathbf{y}_{c}+\boldsymbol{\omega}_{c}\right)>\Gamma\left(\mathbf{y}_{m}, \mathbf{p}_{c}^{*},\left(I^{h *}\right)\right)$. To prove the other half of this equivalence, choose

$$
\mathbf{b}_{c} \in \underset{\mathbf{x}_{c} \in \Re^{c}}{\arg \min } \mathbf{p}_{c}^{*} \cdot \mathbf{x}_{c} \quad \text { subject to } \quad\left(\mathbf{y}_{m}, \mathbf{x}_{c}\right) \in \mathbf{Z}\left(\mathbf{p}_{c}^{*},\left(I^{h *}\right)\right),
$$

so that $\left(\mathbf{y}_{m}, \mathbf{b}_{c}\right) \in \mathbf{Z}\left(\mathbf{p}_{c}^{*},\left(I^{h *}\right)\right)$ and $\mathbf{p}_{c}^{*} \cdot \mathbf{b}_{c}=\Gamma\left(\mathbf{y}_{m}, \mathbf{p}_{c}^{*},\left(I^{h *}\right)\right)$. Then, if we write $\boldsymbol{\omega}+\mathbf{y}=\left(\mathbf{y}_{m}, \mathbf{b}_{c}\right)+$ $\left(\mathbf{0}_{m}, \mathbf{y}_{c}+\boldsymbol{\omega}_{c}-\mathbf{b}_{c}\right)$, we see that if $\mathbf{p}_{c}^{*} \cdot\left(\mathbf{y}_{c}+\boldsymbol{\omega}_{c}\right)>\Gamma\left(\mathbf{y}_{m}, \mathbf{p}_{c}^{*},\left(I^{h *}\right)\right)$ so that $\left(\mathbf{0}_{m}, \mathbf{y}_{c}+\boldsymbol{\omega}_{c}-\mathbf{b}_{c}\right) \in \mathbf{H}^{+}\left(\mathbf{p}_{c}^{*}\right)$, then $\boldsymbol{\omega}+\mathbf{y} \in \mathbf{Z}^{+}\left(\mathbf{p}_{c}^{*},\left(I^{h *}\right)\right)$.

${ }^{13}$ Boundary problems can occur if a Pareto superior allocation involves no consumption of competitive goods and lies on the boundary of $\mathbf{Q}^{+}$, as in Figure 3.1, Panel A. 
prices $\mathbf{p}_{c}$. Because buyers have quasi-concave preferences and competitive firms have convex technologies, their marginal willingness to pay falls (weakly) as the monopoly tries to sell more. Marginal cost must fall faster than willingness to pay to make the monopoly want to increase output. Similarly, to tempt the monopoly to lower output, its marginal cost must rise as it lowers output faster than marginal willingness to pay does. Decentralization problems occur when the increasing returns in producing the monopoly goods are so dramatic that they overwhelm the decreasing returns of households and competitive firms in using these goods.

\subsection{Two-Part Tariffs}

Although take-it-or-leave-it offers give a PDM maximum flexibility, this section demonstrates that two-part tariffs are usually sufficient. In fact, a PDM can generally maximize profits using a two-part tariff with a uniform per-unit price equal to marginal cost so that all discrimination occurs through hookup charges. The intuition behind this result is that if buyers' marginal valuations differ from each other, then the monopoly can profit by adjusting its marketing strategy without changing its production plan, and if these valuations differ from marginal cost, then the monopoly can profit by changing its production level.

We follow the literature in using the Clarke normal cone to generalize marginal cost for nonconvex and nonsmooth technologies. (See, e.g., Khan and Vohra (1987) and Brown (1991).) This cone reduces to the standard normal cone for convex sets.

Definition 3: The Clarke normal cone to a set $\mathbf{Y} \subset \Re^{m+c}$ at $\mathbf{y} \in \mathbf{Y}$ is the set $\mathbf{N}_{\mathbf{Y}}(\mathbf{y}) \equiv\left\{\mathbf{p} \in \Re^{m+c} \mid \mathbf{p} \cdot \mathbf{z} \leq 0 \forall \mathbf{z} \in \mathbf{T}_{\mathbf{Y}}(\mathbf{y})\right\}$, where the Clarke tangent cone $\mathbf{T}_{\mathbf{Y}}(\mathbf{y})$ is given by $\mathbf{T}_{\mathbf{Y}}(\mathbf{y}) \equiv\left\{\mathbf{z} \in \Re^{m+c} \mid\right.$ for every sequence $\left(\mathbf{y}^{k} \in \mathbf{Y}\right)$, where $\mathbf{y}^{k} \rightarrow \mathbf{y}$, and every sequence $\left(t^{k}>0\right)$, where $t^{k} \rightarrow 0, \exists \mathbf{z}^{k} \rightarrow \mathbf{z}$ such that $\left.\mathbf{y}^{k}+t^{k}\left(\mathbf{z}^{k}\right) \in \mathbf{Y} \forall k\right\}$.

Defintion 4: We say that a PDM equilibrium $\left\langle\left(R^{h *}\right),\left(R^{f *}\right)_{f \neq m},\left(\mathbf{x}^{h *}\right)\right.$, $\left.\left(\mathbf{y}^{f *}\right), \mathbf{p}_{c}^{*}\right\rangle$ can be replicated with two-part tariffs if for each household $h$ and competitive firm $f$ there exist hookups $q^{h}, q^{f} \geq 0$ and per-unit prices $\mathbf{p}_{m}^{h}, \mathbf{p}_{m}^{f} \in$ $\left(\Re_{+} \cup\{x\}\right)^{m}$ such that $R^{h *}=q^{h}+\mathbf{p}_{m}^{h} \cdot \mathbf{x}_{m}^{h *}, R^{f *}=q^{f}-\mathbf{p}_{m}^{f} \cdot \mathbf{y}_{m}^{f *}$,

$$
\begin{array}{ll}
\mathbf{x}^{h *} \in \underset{\mathbf{x} \in \Re_{+}^{m+c}}{\arg \max } U^{h}(\mathbf{x}) \quad \text { subject to } & \mathbf{p}_{c}^{*} \cdot \mathbf{x}_{c}+R^{h}\left(\mathbf{x}_{m}\right) \leq \mathbf{p}_{c}^{*} \cdot \mathbf{x}_{c}^{h *}+R^{h *}, \\
\text { where } R^{h}\left(\mathbf{x}_{m}\right) \equiv \begin{cases}q^{h}+\mathbf{p}_{m}^{h} \cdot \mathbf{x}_{m} & \text { for } \mathbf{x}_{m}>\mathbf{0}_{m}, \\
0 & \text { for } \mathbf{x}_{m}=\mathbf{0}_{m},\end{cases}
\end{array}
$$

and

$$
\mathbf{y}_{m}^{f *} \in \underset{\mathbf{y} \in \mathbf{Y}^{\mathbf{f}}}{\arg \max } \mathbf{p}_{c} \cdot \mathbf{y}_{c}-R^{f}\left(\mathbf{y}_{m}\right),
$$



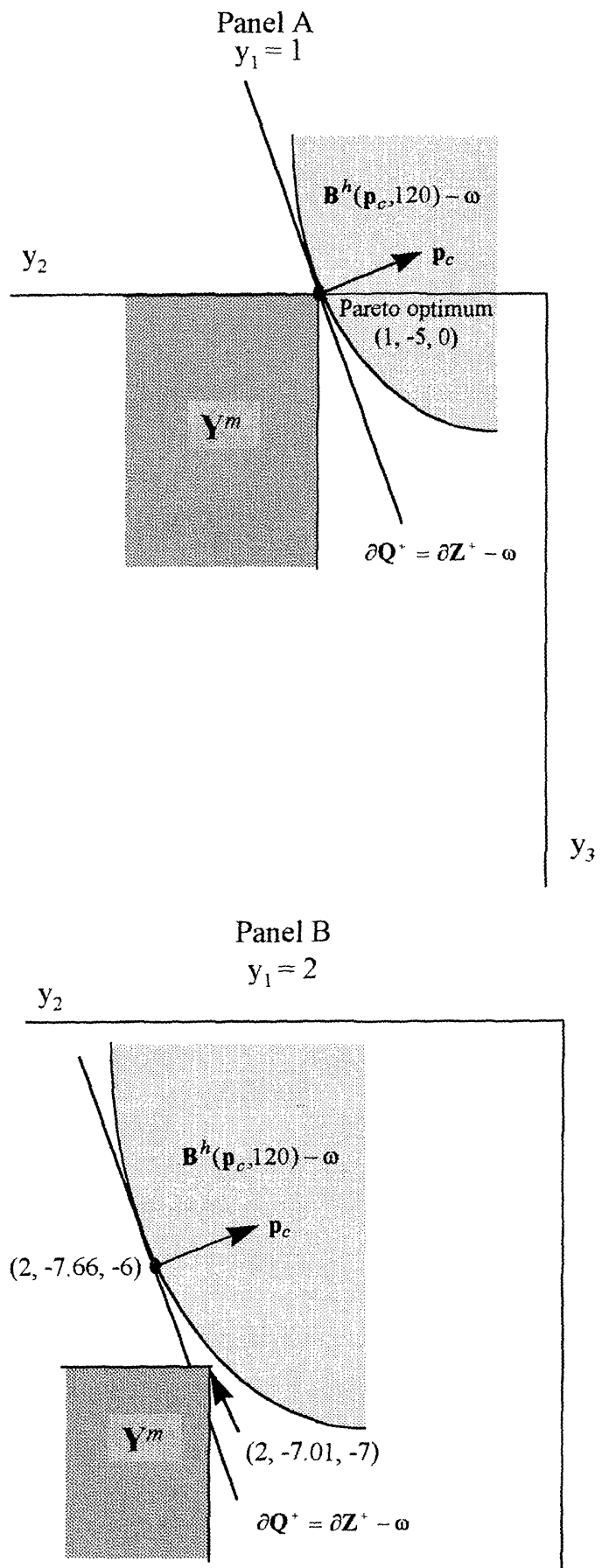

$y_{3}$

Figure 4.2.-The example in Section 3.3. The cross-section in Panel A holds $y_{1}=1$, the Pareto optimal level. Given that $\mathbf{p}_{c}=(9,4)$, the monopoly prefers, however, to produce two units of output, the level held fixed in Panel B's cross-section, because $\mathbf{y}^{m}$ cuts inside $\mathbf{Q}^{+}$. 
where

$$
R^{f}\left(\mathbf{y}_{m}\right) \equiv \begin{cases}q^{f}-\mathbf{p}_{m}^{f} \cdot \mathbf{y}_{m} & \text { for } \mathbf{y}_{m}<\mathbf{0}_{m} \\ 0 & \text { for } \mathbf{y}_{m}=\mathbf{0}_{m}\end{cases}
$$

Allowing infinite per-unit prices gives the monopoly the option of refusing to sell a good. Requiring nonnegative hookups imposes only a slight restriction in the above definition, since negative hookups imply that buyers can improve upon their reservation utility or profit levels, which Lemma 3 shows is typically inconsistent with the PDM maximizing profits.

LEMMA 3: $\Pi^{f}\left(\mathbf{y}_{m}^{f *}, \mathbf{p}_{c}^{*}\right)-R^{f *}=\Pi^{f}\left(\mathbf{0}_{m}, \mathbf{p}_{c}^{*}\right), \quad \forall f \neq m$, in any equilibrium $\left\langle\left(R^{h *}\right),\left(R^{f *}\right)_{f \neq m},\left(\mathbf{x}^{h *}\right),\left(\mathbf{y}^{f *}\right), \mathbf{p}_{c}^{*}\right\rangle$. Moreover, if $(F-1)$ holds, then $\forall h, V^{h}\left(\mathbf{0}_{m}\right.$, $\left.I^{h *}, \mathbf{p}_{c}^{*}\right)=U^{h}\left(\mathbf{x}^{h *}\right)$ where $I^{h *} \equiv R^{h *}+\mathbf{p}_{c}^{*} \cdot \mathbf{x}_{c}^{h *}$.

\section{Proof: See Appendix.}

The idea behind this lemma is that a PDM can generally raise its charge to any household whose utility exceeds its reservation utility or to any firm whose profit exceeds its reservation profit. The only difficulty occurs when a household spends all of its money on the monopoly goods. In that case, a household's equilibrium utility, $U^{h}\left(\mathbf{x}^{h *}\right)$, might exceed its reservation utility $V^{h}\left(\mathbf{0}_{m}, I^{h *}, \mathbf{p}_{c}^{*}\right)$ if (F-1) fails and no household consumed competitive goods. (Consider, e.g., the equilibrium $\left\langle R^{h}=1, \mathbf{x}^{h}=(2,0), \mathbf{y}^{m}=(2,-1), p_{c}=1\right\rangle$ in the economy in Section 3.1.)

Marginal cost must be positive if the per-unit price equals marginal cost, or else demand would be infinite. Assumption (F-1), however, is not quite strong enough to guarantee positive marginal cost, as we can see by modifying the example in Section 3.1, letting $\mathbf{Y} \equiv\left\{\left(x_{1}, x_{2}\right) \mid x_{1} \geq 0, x_{2}=-\frac{1}{8}\left(x_{1}-2\right)^{3}-1\right\}-\mathfrak{R}_{+}^{2}$, $\boldsymbol{\omega} \equiv(0,1)$ and $U\left(x_{1}, x_{2}\right) \equiv x_{1}+x_{2}$. Although this technology satisfies (F-1), marginal cost is 0 when $\mathbf{y}=(2,-1)$, and hence the equilibrium $\langle R=2, \mathbf{x}=(2,0)$, $\left.\mathbf{y}=(2,-1), p_{c}=1\right\rangle$ depicted in Figure 4.3 cannot be replicated with two-part tariffs. Consider, therefore, the following variant of Vohra's (1992) bounded marginal returns assumption:

$(\mathrm{F}-1)^{\prime}$ For all $\mathbf{y}^{m} \in \mathbf{Y}^{m}, \mathbf{y}_{m}^{m}>\mathbf{0}_{m}$, and for all $\mathbf{z}_{m}<\mathbf{0}_{m}$ for which $\mathbf{y}_{m}^{m}+\mathbf{z}_{m} \geq \mathbf{0}_{m}$, there exists $\mathbf{z}_{c} \in \mathfrak{R}^{c}, \mathbf{z}_{c}>\mathbf{0}_{c}$, satisfying:

for all sequences $\mathbf{y}^{q} \rightarrow \mathbf{y}^{m}$, where $\mathbf{y}^{q} \in \mathbf{Y}^{m}$, and sequences $t^{q} \rightarrow 0$, where $t^{q} \in(0, \infty), \mathbf{y}^{q}+t^{q}\left(\mathbf{z}_{m}, \mathbf{z}_{c}\right) \in \mathbf{Y}^{m}$ for $q$ sufficiently large.

If some monopoly good is produced at $\mathbf{y}^{m} \in \mathbf{Y}^{m}$, assumption (F-1)' says that there is a nonzero rate of trading off this good for competitive goods, and that this tradeoff is possible both at and near $\mathbf{y}^{m}$. This assumption is stronger than (F-1), and eliminates the sort of technology depicted in Figure 4.3, ensuring that the monopoly goods are costly at the margin. 


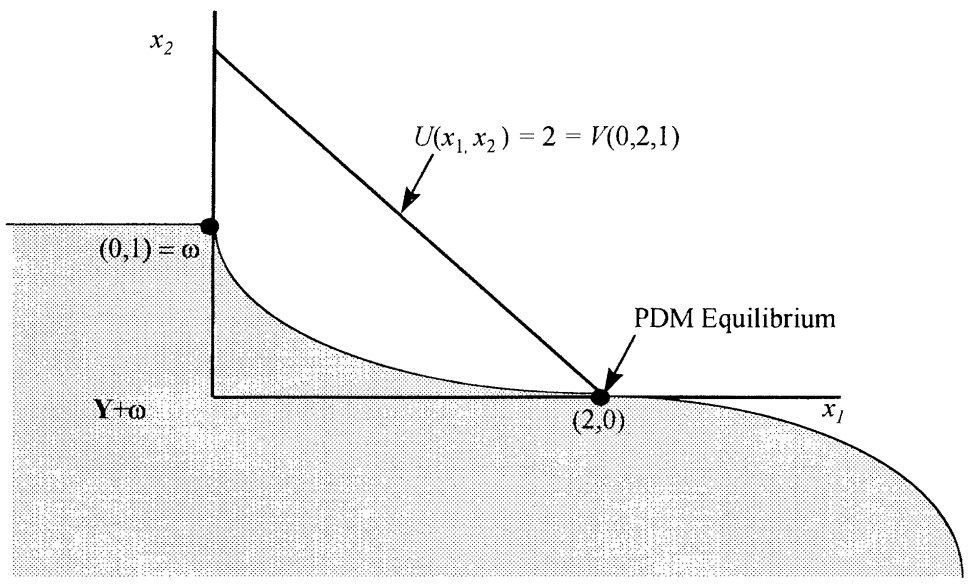

FIGURE 4.3.-Equilibrium that cannot be replicated by two-part tariffs.

Theorem 4.1 below shows that the monopoly can charge per-unit prices in the Clarke normal cone for the goods it wants to sell, although the prices on the goods it does not sell could potentially exceed cost. ${ }^{14}$ To be precise, suppose that the competitive prices are $\mathbf{p}_{c}$ and the monopoly produces $\mathbf{y}^{m} \in \mathbf{Y}^{m}$ with $\hat{m}$ of the monopoly goods produced in positive quantity. Reorder the monopoly goods so that $y_{i}^{m}=0$ for $i=1, \ldots, m-\hat{m}$, and $y_{i}^{m}>0$ for $i=m-\hat{m}+1, \ldots, m$, and let $\hat{\mathbf{y}}^{m}$ denote the last $\hat{m}+c$ components of $\mathbf{y}^{m}$. Then, we say that the monopoly is selling at a uniform per-unit price equal to marginal cost if it charges two-part tariffs with the same per-unit price $\mathbf{p}_{\hat{m}} \in \mathfrak{R}^{\hat{m}}$ for each household and firm, and if

$$
\left(\mathbf{p}_{\hat{m}}, \mathbf{p}_{c}\right) \in \mathbf{N}_{\hat{\mathbf{Y}}^{m}}\left(\hat{\mathbf{y}}^{m}\right) \quad \text { where } \quad \hat{\mathbf{Y}}^{m} \equiv\left\{\mathbf{z} \in \Re^{\hat{m}+c} \mid\left(\mathbf{0}_{m-\hat{m}}, \mathbf{z}\right) \in \mathbf{Y}^{m}\right\} .
$$

\section{THEOREM 4.1 (Two-Part-Tariff Theorem): Any PDM equilibrium}

$$
\left\langle\left(R^{h *}\right),\left(R^{f *}\right)_{f \neq m},\left(\mathbf{x}^{h *}\right),\left(\mathbf{y}^{f *}\right), \mathbf{p}_{c}^{*}\right\rangle
$$

can be replicated with two-part tariffs for which the monopoly is selling at a uniform per-unit price equal to marginal cost if $(F-1)$ holds. ${ }^{15}$

Proof: Suppose that (F-1)' holds, and consider a PDM equilibrium $\left\langle\left(R^{h *}\right)\right.$, $\left.\left(R^{f *}\right)_{f \neq m},\left(\mathbf{x}^{h *}\right),\left(\mathbf{y}^{f *}\right), \mathbf{p}_{c}^{*}\right\rangle$, with incomes $I^{h *}$ defined as in Definition 1. If $\mathbf{y}_{m}^{m *}=\mathbf{0}_{m}$, then $R^{h *}=0$ and $R^{f *}=0 \forall h, f \neq m$, and the theorem is trivial: the equilibrium can be replicated by setting $\mathbf{p}_{m}=(\infty, \infty, \ldots, \infty)$. So assume $\mathbf{y}_{m}^{m *} \neq \mathbf{0}_{m}$ and let $\hat{m}$ be the number of monopoly goods produced in positive quantity.

\footnotetext{
${ }^{14}$ It seems unimportant to search for conditions under which goods that are not sold can be offered at cost without risk of being bought.

${ }^{15} \mathrm{We}$ are grateful to one of our anonymous referees for pointing out the connection between bounded marginal returns and the Two-Part-Tariff Theorem.
} 
We begin by modifying economy $E$ to form $\hat{E}$, an economy with $F+1$ firms, the $\hat{m}$ monopoly goods actually produced in $E$, and the $c$ competitive goods. Reorder the goods as in the discussion above, so that $y_{i}^{m *}=0$ for $i=1, \ldots, m-$ $\hat{m}$ and $y_{i}^{m *}>0$ for $i=m-\hat{m}+1, \ldots, m$. Define

$$
\begin{aligned}
& \hat{\mathbf{Y}}^{f} \equiv\left\{\mathbf{y} \in \Re^{\hat{m}+c} \mid\left(\mathbf{0}_{m-\hat{m}}, \mathbf{y}\right) \in \mathbf{Y}^{f}\right\}, \quad f=1, \ldots, F, \\
& \hat{\mathbf{Y}}^{F+1} \equiv\left\{\mathbf{y} \in \Re^{\hat{m}+c} \mid\left(\mathbf{0}_{m-\hat{m}}, \mathbf{y}\right) \in \mathbf{H}\left(\mathbf{p}_{c}^{*}\right)\right\}-\Re_{+}^{\hat{m}+c}, \\
& \hat{U}^{h}(\mathbf{x}) \equiv U^{h}\left(\mathbf{0}_{m-\hat{m}}, \mathbf{x}\right), \forall \mathbf{x} \in \Re_{+}^{\hat{m}+c}, \quad h=1, \ldots, H, \quad \text { and } \\
& \hat{\mathbf{B}}^{h}\left(\mathbf{p}_{c}, I^{h}\right) \equiv\left\{\mathbf{x} \in \Re_{+}^{\hat{m}+c} \mid \hat{U}^{h}(\mathbf{x}) \geq V^{h}\left(\mathbf{0}_{m}, I^{h}, \mathbf{p}_{c}\right)\right\}, \quad h=1, \ldots, H .
\end{aligned}
$$

Let $\hat{\mathbf{x}}^{h *}, \hat{\boldsymbol{\omega}}^{h}$, and $\hat{\mathbf{y}}^{f *}$ be the last $\hat{m}+c$ components of $\mathbf{x}^{h *}, \boldsymbol{\omega}^{h}$, and $\mathbf{y}^{f *}$ respectively, and let $\hat{\mathbf{y}}^{F+1 *} \equiv \mathbf{0}_{\hat{m}+c}$. Observe that $\left\langle\left(R^{h *}\right),\left(R^{f *}\right)_{f \neq m},\left(\hat{\mathbf{x}}^{h *}\right)\right.$, $\left.\left(\hat{\mathbf{y}}^{f *}\right)_{f=1}^{F+1}, \mathbf{p}_{c}^{*}\right\rangle$ is a PDM equilibrium for the economy $\hat{E} \equiv\left\langle\left(\hat{\boldsymbol{\omega}}^{h}\right),\left(\hat{U}^{h}\right),\left(\hat{\mathbf{Y}}^{f}\right)\right.$, $\left.\left(\theta^{h f}\right)\right\rangle$ and that $(\mathrm{F}-1)^{\prime}$ must hold for $\hat{E}^{16}$ Hence, Theorem 3.1 implies that $\left(\hat{\mathbf{x}}^{h *}\right),\left(\hat{\mathbf{y}}^{f *}\right)$ is a Pareto optimum in economy $\hat{E}$, and so by Khan and Vohra's (1987, Theorem 1) Generalized Second Welfare Theorem, ${ }^{17}$ there exists $\hat{\mathbf{p}} \neq \mathbf{0}_{\hat{m}+c}$ such that

$$
\begin{aligned}
& \hat{\mathbf{p}} \in \mathbf{N}_{\hat{\mathbf{Y}}^{f}}\left(\hat{\mathbf{y}}^{f *}\right), \quad \forall f, \quad \text { and } \\
& -\hat{\mathbf{p}} \in \mathbf{N}_{\hat{\mathbf{B}}^{h}\left(\mathbf{p}_{c}^{*}, I^{h *}\right)}\left(\hat{\mathbf{x}}^{h *}\right), \quad \forall h .
\end{aligned}
$$

Because of the construction of $\hat{\mathbf{Y}}^{F+1}$, expression (a) implies that $\hat{\mathbf{p}}_{c}=\alpha \mathbf{p}_{c}^{*}$ for some $\alpha .{ }^{18}$ Since strict monotonicity implies that $\mathbf{p}_{c}^{*} \gg \mathbf{0}_{c}$, either $\alpha>0$ and $\hat{\mathbf{p}}_{c} \gg \mathbf{0}_{c}$, or $\alpha=0$ and $\hat{\mathbf{p}}_{c}=\mathbf{0}_{c}$.

To use $\hat{\mathbf{p}}$ as the basis for a two-part tariff, we must first show that $\hat{\mathbf{p}}_{c} \gg \mathbf{0}_{c}$. Observe that if $\hat{\mathbf{p}}_{c}=\mathbf{0}_{c}$, then $\hat{p}_{i}>0$ for some $i \in\{1, \ldots, \hat{m}\}$; moreover, since $\hat{\mathbf{y}}^{m *} \gg \mathbf{0}_{m}$, some agent buys good $i$. But, if some household $t$ does, so that $\hat{x}_{i}^{\iota *}>0$, then strict monotonicity and expression (b) imply that $\hat{\mathbf{p}} \gg \mathbf{0}_{\hat{m}+c}$. On the other hand, if some competitive firm $t$ buys good $i$, so that $\hat{y}_{i}^{t *}<0$, then expression (a) implies that $\hat{p}_{s}>0$ for some competitive good $s>\hat{m} .{ }^{19}$ In either event, $\hat{\mathbf{p}}_{c} \neq \mathbf{0}_{c}$, so $\alpha>0$ and $\hat{\mathbf{p}}_{c} \gg \mathbf{0}_{c}$.

We next show that $\hat{\mathbf{p}}_{\hat{m}} \gg \mathbf{0}_{\hat{m}}$. Consider any $i \in\{1, \ldots, \hat{m}\}$ and the vector $\mathbf{e}_{i} \in \Re^{\hat{m}}$ with $y_{i}^{m *}$ in the $i$ th component and "0's" elsewhere. Since (F-1)" holds for $\hat{\mathbf{Y}}^{m}, \exists \mathbf{z}_{c} \in \mathfrak{R}^{c}, \mathbf{z}_{c}>\mathbf{0}_{c}$, satisfying: for all sequences $\mathbf{y}^{q} \rightarrow \hat{\mathbf{y}}^{m *}$, where $\mathbf{y}^{q} \in \hat{\mathbf{Y}}^{m}$, and sequences $t^{q} \rightarrow 0$, where $t^{q} \in(0, \infty), \mathbf{y}^{q}+t^{q}\left(-\mathbf{e}_{i}, \mathbf{z}_{c}\right) \in \hat{\mathbf{Y}}^{m}$ for $q$ sufficiently

\footnotetext{
${ }^{16}$ Note that $\hat{\mathbf{Y}}^{F+1}$ was constructed so that $\forall \mathbf{y} \in \hat{\mathbf{Y}}^{F+1}, \mathbf{p}_{c}^{*} \cdot \mathbf{y}_{c} \leq 0$, so that firm $F+1$ makes no profit.

${ }^{17}$ To apply their theorem, we must also observe that $\hat{\mathbf{Y}}^{f}-\mathfrak{R}_{+}^{\hat{m}+c} \subseteq \hat{\mathbf{Y}}^{f} \forall f$, and that preferences $\hat{U}^{h}(\cdot)$ are continuous, strictly monotonic, and quasi-concave for all $h$.

${ }^{18}$ To see this, note that $\hat{\mathbf{p}}_{c}$ can be decomposed into the sum of a component parallel to $\mathbf{p}_{c}^{*}$ and a component orthogonal to $\mathbf{p}_{c}^{*}$ : i.e., $\hat{\mathbf{p}}_{c}=\alpha \mathbf{p}_{c}^{*}+\mathbf{h}_{c}$ for some $\alpha \in \Re$ and $\mathbf{h}_{c}$ where $\mathbf{h}_{c} \cdot \mathbf{p}_{c}^{*}=0$. Thus $\hat{\mathbf{p}}_{c} \cdot \mathbf{h}_{c}=\mathbf{h}_{c} \cdot \mathbf{h}_{c}$. If $\mathbf{h}_{c}=\mathbf{0}_{c}$, then $\hat{\mathbf{p}}_{c}=\alpha \mathbf{p}_{c}^{*}$, as claimed. If $\mathbf{h}_{c} \neq \mathbf{0}_{c}$, then $\hat{\mathbf{p}}_{c} \cdot \mathbf{h}_{c}>0$; but this contradicts (a) since $\left(\mathbf{0}_{\hat{m}}, \mathbf{h}_{c}\right) \in \hat{\mathbf{Y}}^{F+1}, \hat{\mathbf{Y}}^{F+1}$ is convex, and $\hat{\mathbf{y}}^{F+1 *}=\mathbf{0}_{\hat{m}+c}$.

${ }^{19}$ Otherwise $\hat{\mathbf{p}} \cdot \hat{\mathbf{y}}^{\prime *}<0$, which contradicts (a) since $\mathbf{Y}^{t}$ is convex and $\mathbf{0}_{\hat{m}+c} \in \mathbf{Y}^{t}$.
} 
large. Choosing $\mathbf{z}^{q} \equiv\left(-\mathbf{e}_{i}, \mathbf{z}_{c}\right)$ for $q$ sufficiently large and $\mathbf{z}^{q} \equiv \mathbf{0}_{\hat{m}+c}$ otherwise shows that $\left(-\mathbf{e}_{i}, \mathbf{z}_{c}\right) \in \mathbf{T}_{\hat{\mathbf{y}}^{m}}\left(\hat{\mathbf{y}}^{m *}\right)$. Hence, (a) implies that $\hat{\mathbf{p}} \cdot\left(-\mathbf{e}_{i}, \mathbf{z}_{c}\right) \leq 0$, and since $\hat{\mathbf{p}}_{c} \cdot \mathbf{z}_{c}>0, \hat{p}_{i}>0$. Repeating this argument for $i=1, \ldots, \hat{m}$ shows that $\hat{\mathbf{p}}_{\hat{m}} \gg \mathbf{0}_{\hat{m}}$.

Consider prices $\hat{\mathbf{p}}^{*} \equiv \hat{\mathbf{p}} / \alpha$, and note that $\hat{\mathbf{p}}_{c}^{*}=\mathbf{p}_{c}^{*}$. Define hookups

$$
\begin{aligned}
& q^{h *} \equiv I^{h *}-\hat{\mathbf{p}}^{*} \cdot \hat{\mathbf{x}}^{h *}, \quad \text { and } \\
& q^{f *} \equiv \hat{\mathbf{p}}^{*} \cdot \hat{\mathbf{y}}^{f *}-\Pi^{f}\left(\mathbf{0}_{m}, \mathbf{p}_{c}^{*}\right), \quad \forall h, \quad f \neq m .
\end{aligned}
$$

Consider the $(m+c)$-tuple $\mathbf{p}^{*} \equiv\left(\infty, \infty, \ldots, \infty, \hat{\mathbf{p}}^{*}\right)$ and suppose the monopoly charges the per-unit price $\mathbf{p}_{m}^{*}$ and hookups $\left(q^{h *}\right),\left(q^{f *}\right)$. Then, $\mathbf{x}^{h *}$ and $\mathbf{y}^{f *}$ must solve the optimization problems of each household $h$ and competitive firm $f$, since $\mathbf{p}^{*} \gg \mathbf{0}$ and expressions (a) and (b) hold. By construction, $q^{h *}+\mathbf{p}_{m}^{*} \cdot \mathbf{x}_{m}^{h *}=$ $I^{h *}-\mathbf{p}_{c}^{*} \cdot \mathbf{x}_{c}^{*}=R^{h *} \forall h$, and by construction and Lemma $3, q^{f *}-\mathbf{p}_{m}^{*} \cdot \mathbf{y}_{m}^{f *}=R^{f *}$ $\forall f \neq m$. Moreover, expression (a) implies that the monopoly is selling at marginal cost, and Lemma 3 implies that $q^{h *} \geq 0$ and $q^{f *} \geq 0 \forall h, f \neq m$. Hence, the equilibrium can be replicated with two-part tariffs for which the monopoly is selling at a uniform per-unit price equal to marginal cost.

Q.E.D.

REMARK 1: If $\mathbf{y}_{m}^{m} \gg \mathbf{0}_{m}$, then the conclusion in Theorem 4.1 can be strengthened so that $\left(\mathbf{p}_{m}, \mathbf{p}_{c}\right) \in \mathbf{N}_{\mathbf{Y}^{f}}\left(\mathbf{y}^{f *}\right) \forall f$.

Assumption (F-1)' ensures that duality holds so that Hicksian and Marshallian demands are equal. Without some assumption like this one, Arrow's anomalous case might occur as in Figure 4.3, and preclude replication with two-part tariffs. This problem might occur in partial equilibrium as well, but its relevance to the possibility of using two-part tariffs for perfect price discrimination was not noted previously to our knowledge. The duality issue is similar to the problem in the inefficiency example in Section 3.1. There, the PDM had a marginal cost of zero, and produced a quantity equal to the consumer's Hicksian demand evaluated at a price of zero and the consumer's reservation utility level. Since the consumer was not satiated with the monopoly good, the Marshallian demand did not equal the Hicksian, however, indicating that extra production was efficient.

\section{EXISTENCE AND DECENTRALIZATION: SPECIAL CASES}

Decentralization and existence problems typically arise from a combination of having (i) multiple competitive goods, and (ii) a monopoly technology whose nonconvexities are not solely from setup costs. Below, we show that these problems disappear when either of these two factors is absent. Finding an equilibrium in a single competitive good economy amounts to solving a maximization problem. In an economy with a pure fixed cost, however, a fixed-point argument is necessary. 
Definition 5: A candidate equilibrium $\left\langle\left(R^{h *}\right),\left(R^{f *}\right)_{f \neq m},\left(\mathbf{x}^{h *}\right),\left(\mathbf{y}^{f *}\right), \mathbf{p}_{c}^{*}\right\rangle$ is said to be a PDM equilibrium with transfers if the monopoly strategy $\left(\left(\mathbf{x}_{m}^{h *}, R^{h *}\right),\left(\mathbf{y}_{m}^{f *}, R^{f *}\right)_{f \neq m}, \mathbf{y}_{c}^{m *}\right)$ solves [M] given prices $\mathbf{p}_{c}^{*}$ and incomes $I^{h *}=$ $R^{h *}+\mathbf{p}_{c}^{*} \cdot \mathbf{x}_{c}^{h *} \forall h$.

The transfers needed to implement such an equilibrium must sum to zero since total household income equals the sum of profits and the endowment value.

A Pareto optimum $\left(\hat{\mathbf{x}}^{h}\right),\left(\hat{\mathbf{y}}^{f}\right)$ can only be decentralized as an equilibrium with transfers if every household $h$ can achieve utility $U^{h}\left(\hat{\mathbf{x}}^{h}\right)$ without monopoly goods, or if (F-1) fails. ${ }^{20}$ Hence, we shall assume the following.

(H-1) $\forall h, \forall \hat{\mathbf{x}}^{h} \in \Re_{+}^{m+c}$, there exists $\mathbf{x}_{c}^{h} \in \Re_{+}^{c}$ such that $U^{h}\left(\mathbf{0}_{m}, \mathbf{x}_{c}^{h}\right) \geq U^{h}\left(\hat{\mathbf{x}}^{h}\right)$.

\subsection{Economies with a Single Competitive Good}

A PDM controls all the relative prices in economies with a single competitive good, and when $c=1$, the stack of $c-1$ dimensional hyperplanes that form the boundary of $\mathbf{Z}$ becomes simply a stack of points coinciding exactly with the boundary of $\sum \mathbf{B}^{h}-\sum_{f \neq m} \mathbf{Y}^{f}$. Consequently, all Pareto optima can be decentralized. For simplicity, we prove this fact in a setting without competitive firms, though they could be added.

Proposition 1: For any Pareto optimum $\left(\hat{\mathbf{x}}^{h}\right), \hat{\mathbf{y}}^{m}$ in an economy $E \equiv\left\langle\left(\boldsymbol{\omega}^{h}\right)\right.$, $\left.\left(U^{h}\right), \mathbf{Y}^{m},\left(\theta^{h m}\right)\right\rangle$ with $c=1$, there exist $\left(\hat{R}^{h}\right), \hat{p}_{c}$ such that $\left\langle\left(\hat{R}^{h}\right),\left(\hat{\mathbf{x}}^{h}\right), \hat{\mathbf{y}}^{m}, \hat{\hat{p}}_{c}\right\rangle$ is a $P D M$ equilibrium with transfers, provided that $(H-1)$ holds.

PROOF: Let $\hat{p}_{c} \equiv 1$ and $\forall h, \hat{R}^{h} \equiv I^{h}-\hat{x}_{c}^{h}$, where $\hat{I}^{h} \equiv \min x_{c}$ subject to $U^{h}\left(\mathbf{0}_{m}, x_{c}\right) \geq U^{h}\left(\hat{\mathbf{x}}^{h}\right){ }^{21}$ Then $\left\langle\left(\hat{R}^{h}\right),\left(\hat{\mathbf{x}}^{h}\right), \hat{\mathbf{y}}^{m}, \hat{p}_{c}\right\rangle$ is a candidate equilibrium since $\hat{\mathbf{y}}^{m}+\boldsymbol{\omega}=\sum_{h} \hat{\mathbf{x}}^{h}$, and $U^{h}\left(\hat{\mathbf{x}}^{h}\right)=U^{h}\left(\hat{\mathbf{x}}_{m}^{h}, \hat{I}^{h}-\hat{R}^{h}\right)=V^{h}\left(\hat{\mathbf{x}}_{m}^{h}, \hat{I}^{h}-\hat{R}^{h}, \hat{p}_{c}\right)=V^{h}\left(\mathbf{0}_{m}\right.$, $\left.\hat{I}^{h}, \hat{p}_{c}\right) \forall h$. The Pareto optimality of $\left(\hat{\mathbf{x}}^{h}\right), \hat{\mathbf{y}}^{m}$ implies that $\mathbf{Y}^{m}$ cannot intersect $\mathbf{Q}^{+} \equiv \sum \mathbf{B}^{h}\left(\hat{p}_{c}, \hat{I}^{h}\right)-\boldsymbol{\omega}+\mathbf{H}^{+}\left(\hat{p}_{c}\right)=\sum \mathbf{B}^{h}\left(\hat{p}_{c}, \hat{I}^{h}\right)-\boldsymbol{\omega}$ and so the strategy $\left\langle\left(\hat{R}^{h}\right.\right.$, $\left.\left.\hat{\mathbf{x}}_{m}^{h}\right), \hat{y}_{c}^{m}\right\rangle$ must solve [M] according to Lemma 2 . Hence $\left\langle\left(\hat{R}^{h}\right),\left(\hat{\mathbf{x}}^{h}\right), \hat{\mathbf{y}}^{m}, \hat{p}_{c}\right\rangle$ is an equilibrium with transfers.

Q.E.D.

Proposition 2: A PDM equilibrium exists for an economy $E \equiv\left\langle\left(\omega^{h}\right),\left(U^{h}\right)\right.$, $\left.\mathbf{Y}^{m},\left(\theta^{h m}\right)\right\rangle$ if $c=1$ and $(H-1)$ holds.

PROOF: Let $\hat{p}_{c} \equiv 1$ and $\forall \Pi^{m} \in[0, \infty)$ define $\mathbf{B}_{\Pi^{m}} \equiv \sum_{h} \mathbf{B}^{h}\left(\hat{p}_{c}, \omega_{c}^{h}+\theta^{h m} \Pi^{m}\right)$. Figure 5.1 depicts the boundaries of $\mathbf{B}_{\Pi^{m}}$ for various levels of $\Pi^{m}$.

Let $\hat{\Pi}^{m} \equiv \sup \left\{\Pi^{m} \mid\left(\mathbf{Y}^{m}+\boldsymbol{\omega}\right) \cap \mathbf{B}_{\Pi^{m}} \neq \varnothing\right\}$, and observe that $\dot{\hat{\Pi}}^{m}$ is finite because $\mathbf{Y}^{m}+\boldsymbol{\omega}$ is compact and (H-1) holds. (Note that $\hat{\Pi}^{m}$ is $\Pi_{2}^{m}$ in the

${ }^{20}$ Lemma 3 proves this assertion.

${ }^{21}(\mathrm{H}-1)$ implies that $\hat{I}^{h}$ is well defined. 


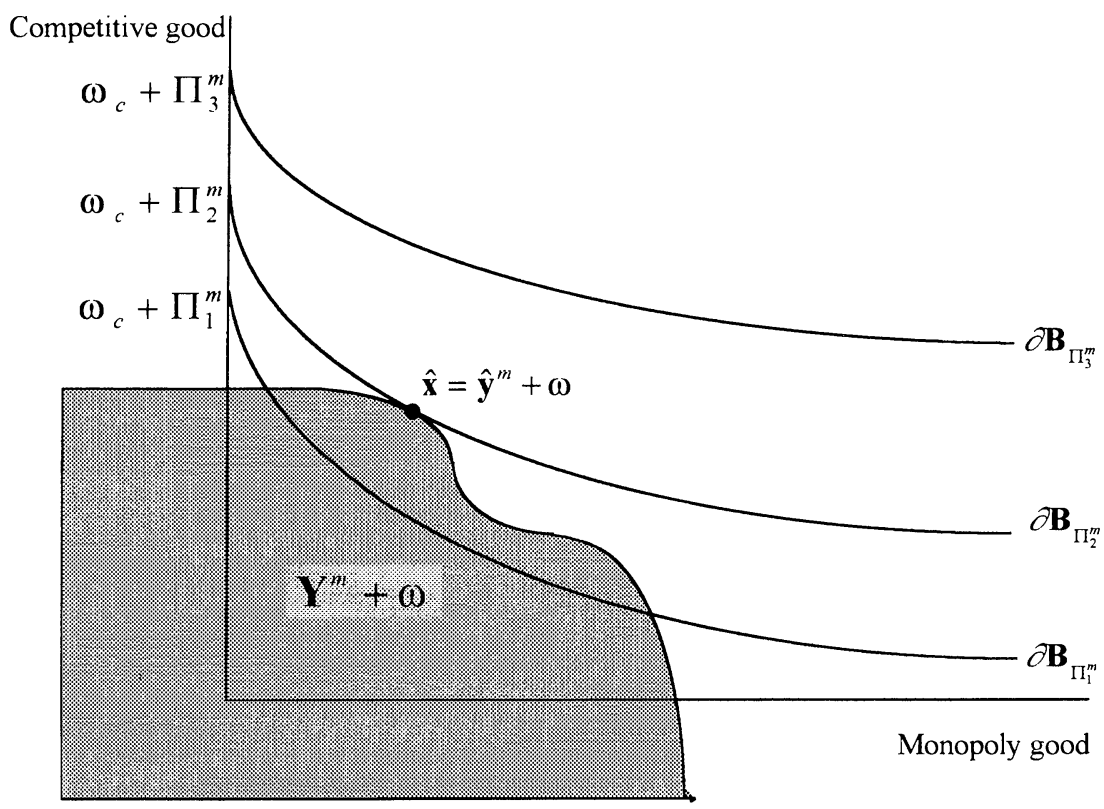

FIGURE 5.1.-Existence in the one competitive good case.

figure.) The construction of $\hat{\Pi}^{m}$ implies that $\mathbf{B}_{\hat{\Pi}^{m}} \cap\left(\mathbf{Y}^{m}+\boldsymbol{\omega}\right) \neq \varnothing,{ }^{22}$ and that there exists a Pareto optimum $\left(\hat{\mathbf{x}}^{h}\right), \hat{\mathbf{y}}^{m}$, where $\hat{\mathbf{x}}^{h} \in \partial \mathbf{B}^{h}\left(\hat{p}_{c}, \omega_{c}^{h}+\theta^{h m} \hat{\Pi}^{m}\right) \forall h$, $\hat{\mathbf{y}}^{m} \in \mathbf{Y}^{m}$ and $\hat{\mathbf{y}}^{m}+\boldsymbol{\omega}=\sum_{h} \hat{\mathbf{x}}^{h}$. Since $\left(\hat{\mathbf{x}}^{h}\right), \hat{\mathbf{y}}^{m}$ is a Pareto optimum, if we define a charge for each household $\hat{R}^{h} \equiv-\hat{x}_{c}^{h}+\min x_{c}$ subject to $U^{h}\left(\mathbf{0}_{m}, x_{c}\right)$ $\geq U^{h}\left(\hat{\mathbf{x}}^{h}\right)$, as in the proof of Proposition 1 , then $\left\langle\left(\hat{R}^{h}\right),\left(\hat{\mathbf{x}}^{h}\right), \hat{\mathbf{y}}^{m}, \hat{p}_{c}\right\rangle$ is an equilibrium with transfers. Moreover, $\forall h, U^{h}\left(\mathbf{0}_{m}, \omega_{c}^{h}+\theta^{h m} \hat{\Pi}^{m}\right)=U^{h}\left(\hat{\mathbf{x}}^{h}\right)$ since $\hat{\mathbf{x}}^{h} \in \partial \mathbf{B}^{h}\left(\hat{p}_{c}, \omega_{c}^{h}+\theta^{h m} \hat{\Pi}^{m}\right)$, and so $\hat{R}^{h}=-\hat{x}_{c}^{h}+\omega_{c}^{h}+\theta^{h m} \hat{\Pi}^{m}$. Hence $\forall h$,

$$
I^{h *} \equiv \omega_{c}^{h}+\theta^{h m}\left(\sum_{\tilde{h}} \hat{R}^{\bar{h}}+y_{c}^{m}\right)=\omega_{c}^{h}+\theta^{h m} \hat{\Pi}^{m}=\hat{R}^{h}+\hat{x}_{c}^{h},
$$

which demonstrates that $\left\langle\left(\hat{R}^{h}\right),\left(\hat{\mathbf{x}}^{h}\right), \hat{\mathbf{y}}^{m}, \hat{p}_{c}\right\rangle$ is an equilibrium.

Q.E.D.

\subsection{Economies with a Pure Fixed Cost}

Existence and decentralization problems may arise when there are multiple competitive goods, but not if the monopoly's increasing returns come solely from

${ }^{22}$ This can be seen by considering any $\hat{\mathbf{x}}$ that is an accumulation point of $\left\{\mathbf{x}_{j}\right\}_{j=1}^{\infty}$, where $\mathbf{x}_{j} \in\left(\mathbf{Y}^{m}+\boldsymbol{\omega}\right) \cap \mathbf{B}_{\Pi_{j}^{m}}, \Pi_{j}^{m} \rightarrow \hat{\Pi}^{m}$. Since $\mathbf{Y}^{m}+\boldsymbol{\omega}$ is closed it contains $\hat{\mathbf{x}}$. Because of the continuity of preferences $\hat{\mathbf{x}} \in \mathbf{B}_{\hat{\Pi}^{m}}$. Therefore $\mathbf{B}_{\hat{\Pi}^{m}} \cap\left(\mathbf{Y}^{m}+\boldsymbol{\omega}\right) \neq \varnothing$. 
setup costs for which there is sufficient willingness to pay. To prove this observation, consider the following pure fixed-cost technology.

(F-2) $\mathbf{Y}^{m}=\{\mathbf{0}\} \cup\left(\{-\boldsymbol{\phi}\}+\mathbf{C}^{m}\right)$ where $\boldsymbol{\phi}_{m}=\mathbf{0}_{m}, \boldsymbol{\omega}_{c}>\boldsymbol{\phi}_{c} \geq \mathbf{0}_{c}$, and $\mathbf{C}^{m}$ is the free disposal hull of a closed convex set containing the origin.

Assumption (F-2) implies that the technology involves a fixed cost $\phi$ followed by decreasing returns. Since $\boldsymbol{\phi}<\boldsymbol{\omega}$, there exist $\left(\boldsymbol{\phi}^{h}\right)$ such that $\boldsymbol{\phi}^{h} \leq \boldsymbol{\omega}^{h} \forall h$ and $\sum_{h} \boldsymbol{\phi}^{h}=\boldsymbol{\phi}$. This allows us to modify $E$ by defining a convex economy $\hat{E} \equiv$ $\left\langle\left(\hat{\boldsymbol{\omega}}^{h}\right),\left(U^{h}\right),\left(\hat{\mathbf{Y}}^{f}\right),\left(\theta^{h m}\right)\right\rangle$, where $\hat{\boldsymbol{\omega}}^{h} \equiv \boldsymbol{\omega}^{h}-\phi^{h}, \hat{\mathbf{Y}}^{m} \equiv \mathbf{C}^{m}$, and $\hat{\mathbf{Y}}^{f} \equiv \mathbf{Y}^{f} \forall f \neq m$. To ensure positive incomes, we use the standard assumption below:

(F-3) $\exists \overline{\mathbf{y}} \in \sum_{f} \hat{\mathbf{Y}}^{f}$ such that $\overline{\mathbf{y}}+\boldsymbol{\omega} \gg 0$.

Lemma 4: For any Pareto optimum $\left(\hat{\mathbf{x}}^{h}\right),\left(\hat{\mathbf{y}}^{f}\right)$ in economy $\hat{E}$, there exist $\left(R^{h}\right)$, $\left(R^{f}\right)_{f \neq m}, \mathbf{p}_{c}$ such that $\left\langle\left(R^{h}\right),\left(R^{f}\right)_{f \neq m},\left(\hat{\mathbf{x}}^{h}\right),\left(\hat{\mathbf{y}}^{f}\right), \mathbf{p}_{c}\right\rangle$ is a PDM equilibrium with transfers in $\hat{E}$, provided that $(H-1),(F-2)$, and $(F-3)$ hold.

Proof: See Edlin, Epelbaum, and Heller (1996).

Q.E.D.

At an equilibrium $\left\langle\left(R^{h}\right),\left(R^{f}\right)_{f \neq m},\left(\hat{\mathbf{x}}^{h}\right),\left(\hat{\mathbf{y}}^{f}\right), \mathbf{p}_{c}\right\rangle$ in $\hat{E}$, we say that there is sufficient willingness to pay the fixed cost $\boldsymbol{\phi}$ if $\mathbf{p}_{c} \cdot \boldsymbol{\phi}_{c}<\hat{\Pi}^{m} \equiv \sum_{h} R^{h}+\sum_{f \neq m} R^{f}$ $+\mathbf{p}_{c} \cdot \hat{\mathbf{y}}_{c}^{m}$. Likewise, we say that there is sufficient willingness to pay at a Pareto optimum in $\hat{E}$ if there is sufficient willingness to pay at the equilibrium that results from decentralizing the optimum.

Proposition 3: For any Pareto optimum $\left(\mathbf{x}^{h}\right),\left(\mathbf{y}^{f}\right)$ in E, with $\mathbf{y}_{m}^{m}>\mathbf{0}_{m}$, there exist $\left(R^{h}\right),\left(R^{f}\right), \mathbf{p}_{c}$ such that $\left\langle\left(R^{h}\right),\left(R^{f}\right)_{f \neq m},\left(\mathbf{x}^{h}\right),\left(\mathbf{y}^{f}\right), \mathbf{p}_{c}\right\rangle$ is a PDM equilibrium with transfers, provided that $(H-1),(F-2)$, and $(F-3)$ hold, and there is sufficient willingness to pay at $\left(\hat{\mathbf{y}}^{f}\right),\left(\mathbf{x}^{h}\right)$ in $\hat{E}$, where $\hat{\mathbf{y}}^{m} \equiv \boldsymbol{\phi}+\mathbf{y}^{m}$ and $\hat{\mathbf{y}}^{f} \equiv \mathbf{y}^{f} \forall f \neq m$.

Proof: See Edlin, Epelbaum, and Heller (1996). Q.E.D.

In order to ensure that every equilibrium in $\hat{E}$ corresponds to an equilibrium in $E$, we make a stronger assumption about endowments:

(F-4) $\boldsymbol{\omega}^{h}>\boldsymbol{\phi} \theta^{h m} \forall h$.

Redefine $\hat{E}$ such that $\hat{\boldsymbol{\omega}}^{h} \equiv \boldsymbol{\omega}^{h}-\boldsymbol{\phi} \theta^{h m}$, and observe that Lemma 4 must hold for this economy as well.

LEMMA 5: A PDM equilibrium exists in $\hat{E}$ if (H-1), (F-2), (F-3), and (F-4) hold.

A Negishi (1960) style argument together with Lemma 4 can be used to prove Lemma 5. We leave this to the reader, who might find it useful to read Edlin, Epelbaum, and Heller (1995, Theorem 7). 
Proposition 4: A PDM equilibrium exists in $E$ if some equilibrium in $\hat{E}$ has sufficient willingness to pay for $\boldsymbol{\phi}$.

Proof: See Edlin, Epelbaum, and Heller (1996).

Q.E.D.

Such existence results would not hold under linear pricing, as Roberts and Sonnenschein (1977) show.

\subsection{Discussion}

The previous two subsections are closely related to important special cases from the regulatory two-part tariff literature. Vohra (1990, Section 5) showed that in economies with only two goods (a monopoly good and a competitive good), there exists an efficient regulated two-part-tariff equilibrium if the technology is of the pure fixed cost type. Moriguchi (1996) has shown under a willingness-to-pay assumption that there exists an efficient regulated two-parttariff equilibrium for pure fixed cost economies. To show the existence of a PDM equilibrium in the fixed cost case, we likewise needed a willingness-to-pay assumption; our assumption was over a smaller set of potential equilibria, but for this we "paid" by making an added assumption about endowments (F-4).

One might hope that Theorem 4.1 would allow us to compare more directly our results with Vohra's and Moriguchi's since it asserts that a PDM can typically charge two-part tariffs. However, the income distribution differs between regulated and deregulated models. In the regulatory models, the monopoly makes no profit, and so to convert a PDM equilibrium into a regulated equilibrium, we would need to lower each household's hookup by its share in monopoly profits. This process might yield hookups of various signs, however, which would violate Vohra's equilibrium definition. (Vohra emphasizes that an equilibrium definition should not admit hookups of different signs, since that would allow wealth redistribution.) Consequently, the allocation in a PDM equilibrium can frequently only be supported as a two-part-tariff equilibrium with transfers. Likewise, transfers are typically required to convert a regulated equilibrium into a PDM equilibrium. Since the monopoly is typically not extracting all surplus in a regulated equilibrium, hookups must be raised for the conversion, and in order to keep consumption unchanged, the monopoly's new profits must be distributed so that each household receives a dividend equal to the increase in that household's hookup. Even with wealth redistribution, not all two-part tariff equilibria can be decentralized as PDM equilibria, since they may not be compatible with profit maximization. In the fixed cost case, those with positive production will be comparable, however, and this observation provides one way to check the efficiency of such regulated equilibria. ${ }^{23}$

\footnotetext{
${ }^{23}$ To show this fact, one need only increase hookups, distribute shares as described, and then argue that the resulting candidate equilibrium is a PDM equilibrium (see Edlin (1993)).
} 
To summarize, despite the close connections between the known cases where PDM equilibria and regulated two-part tariff equilibria exist and are efficient, these cases are not strictly comparable. Perhaps this fact should not be surprising because the distribution of income differs between the models.

\section{COMPARISONS WITH THE LITERATURE}

This paper found that surplus maximization in monopoly markets is usually efficient in equilibrium, even when the surplus in other markets is ignored. This validates the partial equilibrium result that perfect price discrimination is efficient. Moreover, perfect price discrimination usually can be accomplished with discriminatory two-part tariffs with a uniform per-unit tariff equal to marginal cost.

Our paper has developed a new general equilibrium concept for economies with increasing returns, and we conclude with a few remarks about the relationship between our equilibrium concept and previous general equilibrium concepts. As depicted in Figure 6.1, tradeoffs arise between the efficiency of a given type of equilibria and its distributional flexibility. Competitive equilibria, for instance, are always efficient, but Pareto optima frequently cannot be decentralized when there are increasing returns. At the other extreme, Pareto optima can always be decentralized as marginal-cost-pricing equilibria, but these equilibria are frequently inefficient, and for some ownership distributions are always inefficient. PDM equilibria lie between these two extremes: they are typically efficient, but more optima can be decentralized as PDM equilibria than as competitive equilibria because $\mathbf{Q}^{+}$lies above the competitive iso-profit set. Regulated two-part-tariff equilibria allow still more optima to be decentralized, but like marginal-cost-pricing equilibria, these equilibria are not always efficient, and for some distributions of ownership are never efficient.

In Figure 6.1, it appears that marginal-cost-pricing equilibria dominate twopart-tariff equilibria since marginal-cost-pricing equilibria allow more distributional flexibility without any apparent efficiency disadvantage. (See Edlin and Epelbaum (1994) for details.) This may, however, be a figment of the fact that the efficiency axis in the figure has only three groupings. A finer order for efficiency might reveal some advantages of two-part-tariff equilibria. One such ordering might be the inclusive order on the sets of economies for which all equilibria are efficient. After all, the inclusive order orders competitive equilibria and PDM equilibria. We do not know whether such an order or any other order reveals advantages of two-part-tariff equilibria, but this seems a fertile area for future research. Similarly, one may wonder whether there exists an equilibrium concept with a reasonable interpretation that dominates our concept by retaining good efficiency properties while adding distributional flexibility. We conjecture that this is possible because the iso-profit set, $\partial \mathbf{Q}^{+}$, generally lies strictly below the set $\sum \mathbf{B}^{h}-\sum_{f \neq m} \mathbf{Y}^{f}$. 


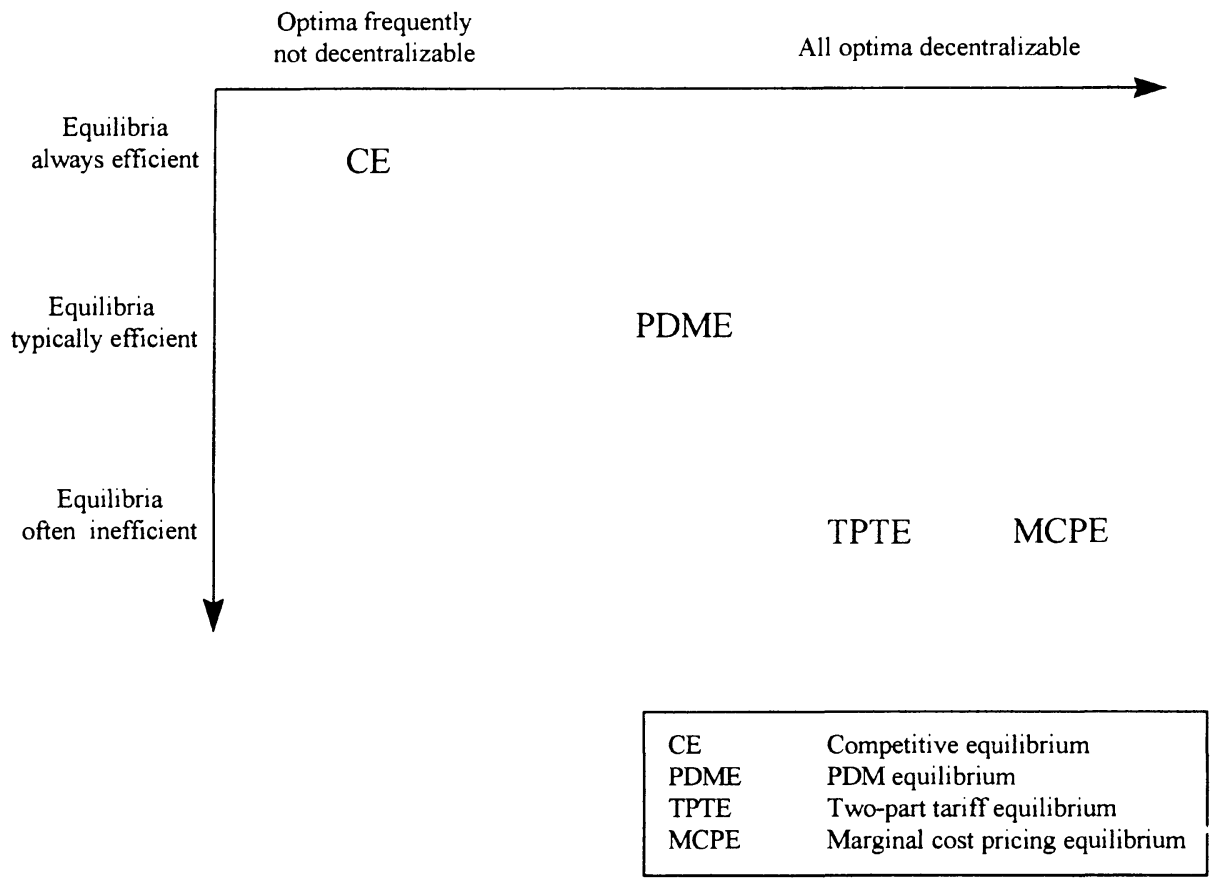

FIGURE 6.1.- Tradeoffs between efficiency and decentralization.

Dept. of Economics, University of California, Berkeley, 549 Evans Hall \#3880, Berkeley, CA 94720, U.S.A.; edlin@econ.berkeley.edu,

Centro de Investigación Económica, Instituto Tecnológico Autónomo de México, Mexico City, Mexico; epelbaum@ms.com,

\section{and}

Dept. of Economics, University of California, San Diego, La Jolla, CA 92093-0508, U.S.A.; wheller@ucsd.edu

Manuscript received August, 1994; final revision received June, 1997.

\section{APPENDIX}

Proof of Lemma 3: Let $\left\langle\left(R^{h *}\right),\left(R^{f *}\right)_{f \neq m},\left(\mathbf{x}^{h *}\right),\left(\mathbf{y}^{f *}\right), \mathbf{p}_{c}^{*}\right\rangle$ be a PDM equilibrium and let $I^{h *} \equiv R^{h *}+\mathbf{p}_{c}^{*} \cdot \mathbf{x}_{c}^{h *} \forall h$. If $\Pi^{f}\left(\mathbf{y}_{m}^{f *}, \mathbf{p}_{c}^{*}\right)-R^{f *}>\Pi^{f}\left(\mathbf{0}_{m}, \mathbf{p}_{c}^{*}\right)$, for some competitive firm $f$, then the monopoly could increase profits by raising its charge $R^{f *}$ to that firm. Hence $\Pi^{f}\left(\mathbf{y}_{m}^{f *}, \mathbf{p}_{c}^{*}\right)-R^{f *}=$ $\Pi^{f}\left(\mathbf{0}_{m}, \mathbf{p}_{c}^{*}\right) \forall f$. Now, suppose that $U^{s}\left(\mathbf{x}^{s *}\right)>V^{s}\left(\mathbf{0}_{m}, I^{s *}, \mathbf{p}_{c}^{*}\right)$ for some $s$ and that $(\mathrm{F}-1)$ holds. If $\mathbf{x}_{m}^{s *}=\mathbf{0}_{m}$, then $R^{s *}<0$ and the monopoly could increase profits simply by raising $R^{s *}$ to 0 . If $\mathbf{x}_{m}^{s *}>\mathbf{0}_{m}$, then the continuity of preferences ensures that $\exists \boldsymbol{\varepsilon}_{m}>\mathbf{0}_{m}$ such that $V^{s}\left(\mathbf{x}_{m}^{s *}-\boldsymbol{\varepsilon}_{m}, I^{s *}-\right.$ $\left.R^{s *}, \mathbf{p}_{c}^{*}\right)>V^{s}\left(\mathbf{0}_{m}, I^{s *}, \mathbf{p}_{c}^{*}\right)$. If (F-1) holds, then the monopoly could increase profits by offering $\left(\mathbf{x}_{m}^{s *}-\varepsilon_{m}, R^{s *}\right)$ to household $s$. Hence, if $(\mathrm{F}-1)$ holds then $V^{h}\left(\mathbf{0}_{m}, I^{h *}, \mathbf{p}_{c}^{*}\right)=U^{h}\left(\mathbf{x}^{h *}\right) \forall h . \quad$ Q.E.D. 


\section{REFERENCES}

ArRow, K. J. (1951): "An Extension of the Basic Theorems of Classical Welfare Economics," Proceedings of the Second Berkeley Symposium on Mathematical Statistics and Probability, ed. by J. Neyman. Berkeley: University of California Press, 507-532.

Beato, P., AND A. Mas-Colell (1983): "Gestion au Coût Marginal et Efficacité de la Production Agrégée: Un Example," Annales de LINSEE, 51, 39-46.

- (1985): "On Marginal Cost Pricing with Given Tax-Subsidy Rules," Journal of Economic Theory, 37, 356-365.

BöHM, V. (1990): "General Equilibrium with Profit Maximizing Oligopolists," University of Mannheim Discussion Paper No. 414/90.

- (1994): "The Foundation of the Theory of Monopolistic Competition Revisited," Journal of Economic Theory, 63, 208-218.

BRown, D. J. (1991): "Equilibrium Analysis with Nonconvex Technologies," in Handbook of Mathematical Economics, Volume IV, ed. by W. Hildenbrand and H. Sonnenschein. New York: North-Holland.

BRown, D. J., AND G. M. HEAL (1979): "Equity, Efficiency, and Increasing Returns," Review of Economic Studies, 46, 571-585.

Brown, D. J., W. P. Heller, AND R. M. STARR (1992): "Two-Part Marginal Cost Pricing Equilibria: Existence and Efficiency," Journal of Economic Theory, 57, 52-72.

COASE, R. H. (1946): “The Marginal Cost Controversy,” Economica, 13, 169-189.

DemsetZ, H. (1968): "Why Regulate Utilities?" Journal of Law and Economics, 11, 55-65.

EdLIN, A. S. (1993): "The Efficient Recovery of Fixed Costs: A Collection of Essays," Ph.D. Dissertation, Stanford University.

Edlin, A. S., AND M. Epelbaum (1994) "Surplus Maximization and Price Discrimination' in General Equilibrium: Part 2," Manuscript.

Edlin, A. S., M. Epelbaum, AND W. P. Heller (1995): "Is Perfect Price Discrimination Really Efficient? Welfare and Existence in General Equilibrium," Burch Working Paper No. B95-9, U.C. Berkeley.

(1996): "Is Perfect Price Discrimination Really Efficient?" Welfare and Existence in General Equilibrium," Burch Working Paper No. B97-24, U.C. Berkeley.

GABSZEWICZ, J. J., AND J. VIAL (1972): "Oligopoly 'A la Cournot' in a General Equilibrium Analysis," Journal of Economic Theory, 4, 381-400.

GrodAL, B. (1984): "Profit Maximizing Behavior in General Equilibrium Models with Imperfect Competition," in Economic Essays, ed. by Akademisk Forlag, 28, 79-90.

GUESNERIE, R. (1975): "Pareto Optimality in Non-Convex Economies," Econometrica, 43, 1-29.

Khan, M. A., AND R. Vohra (1987): "An Extension of The Second Welfare Theorem to Economies with Nonconvexities and Public Goods," Quarterly Journal of Economics, 102, 223-241.

MoRiguCHI, C. (1996): "Two-Part Marginal Cost Pricing in a General Equilibrium Model," Journal of Mathematical Economics, 26, 363-385.

NEGISHI, T. (1960): "Welfare Economics and the Existence of an Equilibrium for a Competitive Economy," Metroeconomica, 12, 92-97.

OI, W. Y. (1971): "A Disneyland Dilemma: Two-Part Tariffs for a Mickey Mouse Monopoly," Quarterly Journal of Economics, 85, 77-90.

QUINZII, M. (1992): Increasing Returns and Efficiency. Oxford: Oxford University Press.

ROBERTS, J., AND H. SonNENSCHEIN (1977): "On the Foundations of the Theory of Monopolistic Competition," Econometrica, 45, 101-113.

SPENCE, A. M. (1976): "Product Selection, Fixed Costs, and Monopolistic Competition," Review of Economic Studies, 43, 217-235.

Vohra, R. (1990): "On the Inefficiency of Two-Part Tariffs," Review of Economic Studies, 57, $415-438$.

- (1992): "Marginal Cost Pricing under Bounded Marginal Returns," Econometrica, 60, 859-876. 